\title{
Nonnormal small jump approximation of infinitely divisible distributions
}

\author{
Zhiyi Chi \\ Department of Statistics \\ University of Connecticut \\ Storrs, CT 06269, USA \\ E-mail: zhiyi.chi@uconn.edu
}

October 2, $2018^{1}$

\begin{abstract}
We consider a type of nonnormal approximation of infinitely divisible distributions that incorporates compound Poisson, Gamma, and normal distributions. The approximation relies on achieving higher orders of cumulant matching, to obtain higher rates of approximation error decay. The parameters of the approximation are easy to fix. The computational complexity of random sampling of the approximating distribution in many cases is of the same order as normal approximation. Error bounds in terms of total variance distance are derived. Both the univariate and the multivariate cases of the approximation are considered.
\end{abstract}

Keywords and phrases. Infinitely divisible; normal approximation; compound Poisson approximation; Gamma approximation; cumulant matching; sampling

2000 Mathematics Subject Classifications: Primary 60E07; Secondary 60G51.

\section{Introduction}

Simulation of infinitely divisible (i.d.) random variables has many applications. In most cases, since closed formulas of i.d. distributions are unavailable, good approximation methods are desired. Normal approximation of i.d. distributions, which was studied in [26] and later developed in [1, 11] in the framework of small jump approximation, has received much attention in the literature $[2,13,16,19,22,23,33]$.

The idea of small jump normal approximation is as follows. Denote by $\lambda$ the Lévy measure of an i.d. random variable $X$. Given $r>0$, decompose $\lambda=\lambda_{r}+\left(\lambda-\lambda_{r}\right)$, such that $\lambda-\lambda_{r} \geq 0$ has finite mass. Correspondingly, $X=X_{r}+\Delta_{r}$, where $X_{r}$ and $\Delta_{r}$ are independent i.d. random variables with Lévy measures $\lambda_{r}$ and $\lambda-\lambda_{r}$, respectively. Then $X_{r}$ is approximated by a Gaussian random variable, while $\Delta_{r}$ is sampled using standard methods for compound Poisson random variables. Presumably, in order for the approximation to have a certain degree of precision, the support of $\lambda_{r}$ should be in a small neighborhood of 0 . The size of the neighborhood is controlled by $r$. For the univariate case, it is natural to set $r$ equal to the maximum jump size [1]. However, for the multivariate case, such use of $r$ can be restrictive. Generally speaking, one can use $r$ to index any tunable quantity, as long as it controls (indirectly) the size of the support of $\lambda_{r}$ [11].

Normal approximation relies on second-order moment matching between $X_{r}$ and a Gaussian random variable, by which we mean the matching of their first and second moments. This is equivalent to second-order cumulant matching. Without specifying details, by certain measures, the error of the approximation in the univariate case is bounded by

$$
C|\kappa|_{3, X_{r}} / \kappa_{2, X_{r}}^{3 / 2}
$$

where $C$ is a universal constant, $\kappa_{2, X_{r}}$ is the second cumulant of $X_{r}$, and for $j \geq 3,|\kappa|_{j, X_{r}}=$ $\int|x|^{j} \lambda_{r}(\mathrm{~d} x)$ is the $j$ th "absolute cumulant" of $X_{r}$ [1]. The best currently available value of $C$

\footnotetext{
${ }^{1}$ Submitted for journal publication on March 28, 2013
} 
is 0.4785 ([28], p. 71). Also, in some symmetric cases, since the third cumulants of $X_{r}$ and the Gaussian random variable are $0,|\kappa|_{3, X_{r}}$ in the bound can be more or less replaced with $|\kappa|_{4, X_{r}}$, while the power of $\kappa_{2, X_{r}}$ is raised to 2 [1]. From the pattern of the bound, one could guess that, if $X_{r}$ and some $Y_{r}$ have the same cumulants of order $1, \ldots, q-1$ with $q \geq 5$, then $X_{r}$ could be approximated by $Y_{r}$ with the error being bounded by

$$
C(r)\left(|\kappa|_{q, X_{r}}+|\kappa|_{q, Y_{r}}\right) / \kappa_{2, X_{r}}^{q / 2},
$$

where $C(r)$ is a near-constant, at least when $r$ is small, or most ideally, a universal constant which may depend on the dimension of $X$ but is not very large. Elementary calculations indicates that in many cases, the above bound vanishes at a genuinely higher rate than the bound for normal approximation as $r \rightarrow 0+$. Since the $q$ th cumulant of a Gaussian random variable is 0 , the above bound, if true, is consistent with the bound for normal approximation.

Even by some rough analysis on characteristic functions, there is good reason to expect that the bound is true for both univariate and multivariate cases. However, before attempting to work out the detail, perhaps one should first ask if such an approximation can possibly be implemented easily. The meaning of the question is twofold. First, the distribution of the approximating random variable should be easy to identify; preferably, it is i.d. Second, the approximating random variable should be easy to sample; preferably, the computational complexity of the sampling is of the same order as the normal approximation. If the answer to the question is positive, then the next question is how large $q$ can be. It can be anticipated that the larger $q$ is, the faster the error of approximation vanishes as $r \rightarrow 0+$. After the answers to the two questions are in place, a wide range of available techniques can potentially be modified to establish the error bound (e.g. [3, 5, 8, 28]).

Clearly, cumulant matching is equivalent to moment matching. Actually, our proof of the above type of bound eventually will be based on moment matching. However, thanks to the LévyKhintchine representation, it is more natural and convenient to consider cumulants than moments. We shall show that it is fairly easy to construct approximating i.d. random variables with matching cumulants up to at least the fourth order, in other words, we can get at least $q=5$. In many important cases, we can get $q=6$, and in the symmetric cases, we can get $q=10$. For the univariate case, the construction is particularly simple. The approximating i.d. random variable is the sum of a compound Poisson random variable and an independent Gaussian random variable, with the former in turn being the sum of a Poisson number of i.i.d. Gamma random variables. Importantly, using algorithms already available [14, 20], the computational complexity of the random sampling for the approximation is universally bounded, so it is of the same order as the random sampling from a normal distribution.

We shall refer to the approximation as Poisson-Gamma-Normal (PGN) approximation, although a longer name like "compound Poisson-Normal approximation with Gamma summands and higher order of cumulant matching" might be more appropriate. We shall bound its error in terms of total variation distance by combining Fourier analysis, Lindeberg method (cf. [6] for a modern application of it), and a device in [1]. The results are nonasymptotic and of the aforementioned type. Asymptotically, when applied to $X_{r}$, the approximation yields substantially higher rate of precision than normal approximation as $r \rightarrow 0+$. Of course, on modern treatments of Poisson, compound Poisson, and normal approximations, there is now an extensive literature, and on Gamma and other types of approximations, there is also a growing literature; see [3, 7, 8, 17, 27, 28, 30] and references therein. However, it appears that there has been little work on using convolutions of different types of simple distributions to improve approximation, in the sense that the error of approximation vanishes at a faster rate asymptotically.

For the multivariate case, the issue of approximation becomes quite more involved, which is a well documented phenomenon [5, 8, 11, 17, 28, 32]. For normal approximation of i.d. distributions, 
several important issues unique to the multivariate case are identified and addressed in [11]. The same issues also arise in the type of approximation considered here and actually become more serious. To address them, we consider a "radial" cumulant matching approach. Its idea is to apply the same cumulant matching method for the univariate case to each radial direction in the Lévy-Khintchine representation, in such a way that, when the approximating Lévy measures and Gaussian measures along different radial directions are "bundled" together, we get a valid multivariate i.d. distribution with desired order of cumulant matching and with the covariance of its Gaussian component being precisely evaluated. Although the approach does not completely resolve the aforementioned issues, it seems to work well in many important cases.

As in the univariate case, we shall prove a similar type of bound for the error of the proposed PGN approximation in terms of total variation distance. On the other hand, the issue of computational complexity needs to be considered more carefully. Recall the approximation is applied to $X_{r}$ in the decomposition $X=X_{r}+\Delta_{r}$. As in the univariate case, the approximating random variable for $X_{r}$ has a compound Poisson component. Unfortunately, this component now can only be sampled by summing a large number of Poisson events. As a result, the computational complexity of the approximation of $X_{r}$ is much greater than normal approximation. However, one has to take into account the computational complexity of the sampling of $\Delta_{r}$. We will argue using an example that although the proposed PGN approximation as a whole has greater computational complexity than normal approximation, asymptotically, as $r \rightarrow 0+$, the two have the same order of complexity. Because the PGN approximation can yield substantially higher rate of convergence, therefore, at least asymptotically, it is worth the extra computation. Note that whereas in [11], the focus is the approximation of the related Lévy processes, our discussion is restricted to i.d. distributions. An extension of PGN approximation to processes will be subject to future work.

In Section 2, we shall set up notation and collect useful facts about i.d. distributions. Sections 3 and 4 consider PGN approximation for univariate i.d. distributions and multivariate i.d. distributions, respectively. The proofs of the main results in these two sections are collected in Section 5 and the proofs of related technical results are collected in Section 6.

\section{Preliminaries}

\subsection{Notation}

Denote $\mathbb{Z}_{+}=\{0\} \cup \mathbb{N}$ and $\mathbb{R}_{+}=[0, \infty)$. If $f(x)$ is a function on $\mathbb{R}^{d}$, where $x=\left(x_{1}, \ldots, x_{d}\right)$, then by $f^{(\alpha)}(x)$ or $\partial^{\alpha} f(x)$ we mean 1) $\alpha=\left(\alpha_{1}, \ldots, \alpha_{d}\right) \in \mathbb{Z}_{+}^{d}$, and 2) $f^{(\alpha)}(x)=\partial_{1}^{\alpha_{1}} \cdots \partial_{d}^{\alpha_{d}} f(x)$, where $\partial_{j}^{k}$ denotes the $k$ th-order partial derivative with respect to $x_{j}$. The order of $\alpha$ is defined to be $|\alpha|=\alpha_{1}+\cdots+\alpha_{d}$. Denote $x^{\alpha}=x_{1}^{\alpha_{1}} \cdots x_{d}^{\alpha_{d}}$ and $\alpha !=\alpha_{1} ! \cdots \alpha_{k} !$. Denote by $\mathscr{S}\left(\mathbb{R}^{d}\right)$ the space of rapidly decreasing function on $\mathbb{R}^{d}$. It is a basic fact that the Fourier transform $h \rightarrow \widehat{h}(t)=\int e^{\mathrm{i}\langle t, x\rangle} h(x) \mathrm{d} x$ is an homeomorphism of $\mathscr{S}\left(\mathbb{R}^{d}\right)$ onto itself ([18], p. 103).

Denote by $\operatorname{sppt}(\nu)$ the support of a measure $\nu$. For two random variables $X$ and $Y$, their total variation distance [3] is denoted by

$$
d_{\mathrm{TV}}(X, Y)=\sup \{\mathbb{P}\{X \in A\}-\mathbb{P}\{Y \in A\}: A \text { measurable }\}
$$

and, if $X, Y \in \mathbb{R}$, their Kolmogorov-Smirnov distance is denoted by

$$
d_{\mathrm{KS}}(X, Y)=\sup \{|\mathbb{P}\{X \leq x\}-\mathbb{P}\{Y \leq x\}|: x \in \mathbb{R}\}
$$

For any i.d. random variable $X \in \mathbb{R}^{d}$, denote by $\psi_{X}$ and $\Psi_{X}$ its characteristic function and 
characteristic exponent, respectively

$$
\psi_{X}(t)=e^{-\Psi_{X}(t)}=\mathbb{E}\left[e^{\mathrm{i}\langle t, X\rangle}\right], \quad t \in \mathbb{R}^{d} .
$$

Let $f_{X}$ be the probability density of $X$. If it exists, then $\psi_{X}=\widehat{f}_{X}$. Denote

$$
\kappa_{\alpha, X}=\left.\partial^{\alpha} \ln \mathbb{E}\left[e^{\langle t, X\rangle}\right]\right|_{t=0}, \quad|\kappa|_{\alpha, X}=\int\left|u^{\alpha}\right| \lambda(\mathrm{d} u) .
$$

The quantity $\kappa_{\alpha, X}$ is known as the $\alpha$ th cumulant of $X$. It is well defined provided that $\mathbb{E}\left[e^{\langle t, X\rangle}\right]<\infty$ for all $t$ in a neighborhood of 0 . Some properties of cumulants can be found in [28]. We shall refer to $|\kappa|_{\alpha, X}$ as the $\alpha$ th absolute cumulant of $X$.

\subsection{Basic assumptions and facts}

Let $X \in \mathbb{R}^{d}$ be i.d. with Lévy measure $\lambda$. We will always assume

$$
\Psi_{X}(t)=\int\left(1+\mathrm{i}\langle t, u\rangle-e^{\mathrm{i}\langle t, u\rangle}\right) \lambda(\mathrm{d} u), \quad t \in \mathbb{R}^{d},
$$

in particular, $X$ has no Gaussian component and $\mathbb{E} X=0$. The assumption causes no loss of generality since, if necessary, we can decompose $X$ as $X^{\prime}+X^{\prime \prime}$, such that $X^{\prime}$ has a Lévy measure satisfying (1) and $X^{\prime \prime}$ is a compound Poisson random variable. Then we can take $X^{\prime}-\mathbb{E} X^{\prime}$ as the new $X$. We will also always assume

$$
\lambda\left(\mathbb{R}^{d}\right)=\infty
$$

Under the assumption, $X$ is not compound Poisson and $\mathbb{P}\{X=x\}=0$ for $x \in \mathbb{R}^{d}$ ([31], Theorem 27.4). It is known that if $d=1$ and $\lambda(\mathbb{R})<\infty$ then $X$ does not admit normal approximation [1]. The assumption excludes the case of lattice valued i.d. random variables, for which Poisson-Charlier approximation has been studied [3, 26].

Recall that for any $a>0, \mathbb{E}\|X\|^{a}<\infty$ if and only if $\int 1\{\|u\|>1\}\|u\|^{a} \lambda(\mathrm{d} u)<\infty([31]$, p. 159-160). If $\lambda$ is a Borel measure on $\mathbb{R}^{d}$ with $\lambda(\{0\})=0$ and $\int\left(\|u\|^{2} \wedge 1\right) \lambda(\mathrm{d} u)<\infty$, then $\lambda$ is the Lévy measure of some i.d. random variable ([31], Theorem 8.1). Also, if $\operatorname{sppt}(\lambda)$ is bounded, then $\mathbb{E}\left[e^{\langle t, X\rangle}\right]<\infty$ for all $t \in \mathbb{R}^{d}$ ([31], Theorem 25.17). By differentiation,

$$
\kappa_{\alpha, X}= \begin{cases}0 & |\alpha|=1 \\ \int u^{\alpha} \lambda(\mathrm{d} u) & |\alpha|>1\end{cases}
$$

for all $\alpha$. It is easy to see that if each $\alpha_{i}$ is even or $\operatorname{sppt}(\lambda) \in \mathbb{R}_{+}^{d}$, then $\kappa_{\alpha, X}=|\kappa|_{\alpha, X}$. Also,

$$
\mathbb{V}(X)=\int u u^{\prime} \lambda(\mathrm{d} u)
$$

and hence $\operatorname{tr}(\mathbb{V}(X))=\int\|u\|^{2} \lambda(\mathrm{d} u)$, where $\operatorname{tr}(A)$ denotes the trace of a square matrix $A$.

Let $f \in C\left(\mathbb{R}^{d}\right)$. For $n \in \mathbb{N}$, let $U_{n}$ be i.d. with $\Psi_{U_{n}}=n^{-1} \Psi_{X}$. If $\operatorname{sppt}(f) \subset \mathbb{R}^{d} \backslash\{0\}$ and is compact, then $n \mathbb{E} f\left(U_{n}\right) \rightarrow \int f \mathrm{~d} \lambda$, which directly follows from the vague convergence of $n \mathbb{P}\left\{U_{n} \in \mathrm{d} x\right\}$ to $\lambda(\mathrm{d} x)$ on $\{x:\|x\|>\epsilon\}$ given $\epsilon>0$ ([4], p. 39); see [21] for detail on vague convergence. The next result, which will be used later, concerns the case where $\operatorname{sppt}(f)$ is not a compact set in $\mathbb{R}^{d} \backslash\{0\}$. When $d=1$ and $f(x)=|x|^{p}$ with $p>2$, the result is established as Lemma 3.1 in [1]. However, as seen from the case $X \sim N(0,1)$, the asserted convergence in general is not true if $f(x)=x^{2}$. 
Proposition 1. Suppose $|f(x)| \leq g(\|x\|)$, where $g \in C\left(\mathbb{R}_{+}\right)$is nondecreasing with $g(t)=o\left(t^{2}\right)$ as $t \rightarrow 0+$. Suppose $\mathbb{E}\|X\|^{2}<\infty$ and one of the following holds, 1) $\mathbb{E} g\left(c\left\|X^{\prime}\right\|\right)<\infty$ for some $c>1$, where $X^{\prime}=X_{1}-X_{2}$, with $X_{i}$ i.i.d. $\sim X$, 2) $\mathbb{E} g(2 c\|X\|)<\infty$ for some $c>1$, or 3) provided $X$ is symmetric, $\mathbb{E} g(\|X\|)<\infty$. Then $f \in L^{1}(\lambda)$ and $n \mathbb{E} f\left(U_{n}\right) \rightarrow \int f \mathrm{~d} \lambda$ as $n \rightarrow \infty$.

\section{Univariate Poisson-Gamma-Normal approximation}

\subsection{Cumulant matching}

For simplicity and without loss of generality, we will only consider two cases 1$) \operatorname{sppt}(\lambda) \subset \mathbb{R}_{+}$and 2) $X$ is symmetric. First, suppose $\operatorname{sppt}(\lambda) \subset \mathbb{R}_{+}$. Given $r>0$, decompose $X=X_{r}+\Delta_{r}$, where $X_{r}$ and $\Delta_{r}$ are independent i.d. random variables such that

$$
\Psi_{X_{r}}(t)=\int\left(1+\mathrm{i} t u-e^{\mathrm{i} t u}\right) \lambda_{r}(\mathrm{~d} u), \quad \text { with } \lambda_{r}(\mathrm{~d} u)=\mathbf{1}\{u<r\} \lambda(\mathrm{d} u) .
$$

Given $p \geq-1$, let $Y_{r}$ be an i.d. random variable with

$$
\Psi_{Y_{r}}(t)=\int_{0}^{\infty}\left(1+\mathrm{i} t u-e^{\mathrm{i} t u}\right) \gamma_{r}(\mathrm{~d} u), \quad \text { with } \gamma_{r}(\mathrm{~d} u)=m(r) u^{p} e^{-u / s(r)} \mathrm{d} u,
$$

where $m(r)>0$ and $s(r)>0$ are constants that need to be determined. Finally, let

$$
T_{r}=Y_{r}+\sigma(r) Z, \quad Z \sim N(0,1) \text { independent of } Y_{r},
$$

where $\sigma(r)>0$ is a constant that needs to be determined.

We shall use $T_{r}+\Delta_{r}$ to approximate $X$, or equivalently, use $T_{r}$ to approximate $X_{r}$. But first, let us point out how easy it is to sample $T_{r}$. Clearly, the issue is the sampling of $Y_{r}$. Since $Y_{r}=U-\mathbb{E} U$, where $U \geq 0$ is i.d. with Lévy density $m(r) \mathbf{1}\{u>0\} u^{p} e^{-u / s(r)}$, and $\mathbb{E} U=\Gamma(p+2) m(r) s(r)^{p+2}$, we only need to consider the computational complexity of the sampling of $U$. If $p=-1$, then $U \sim \operatorname{Gamma}(m(r), s(r))$, the Gamma distribution with shape parameter $m(r)$ and scale parameter $s(r)$. It is known that the sampling of $\operatorname{Gamma}(a, b)$ has universally bounded complexity regardless of $(a, b)$ ([14], p. 407-420). If $p>-1$, then $U \sim \sum_{i=1}^{N} \xi_{i}$, where $N \sim \operatorname{Poisson}(a)$ with $a=$ $\int_{0}^{\infty} m(r) u^{p} e^{-u / s(r)} \mathrm{d} u=\Gamma(p+1) m(r) s(r)^{p+1}$, and $\xi_{i}$ are i.i.d. $\operatorname{Gamma}(p+1, s(r))$ random variables independent of $N$. The sampling of Poisson $(a)$ is known to have universally bounded complexity ([15] or [20], p. 228-241). On the other hand, conditional on $N, U \sim \operatorname{Gamma}(N(p+1), s(r)$ ). Therefore, the sampling of $U$, and hence that of $T_{r}$, has the same order of complexity as the sampling of a normal random variable.

Due to the Lévy-Khintchine representation of $T_{r}$, we refer to the approximation of $X$ by $T_{r}+\Delta_{r}$, or $X_{r}$ by $T_{r}$, as Poisson-Gamma-Normal (PGN) approximation.

It is easy to see $\mathbb{E} X_{r}=\mathbb{E} T_{r}=\mathbb{E} Y_{r}=0$, and for $j \geq 2$,

$$
\begin{gathered}
\kappa_{j, X_{r}}=\int u^{j} \lambda_{r}(\mathrm{~d} u), \quad \kappa_{j, T_{r}}=\kappa_{j, Y_{r}}+\mathbf{1}\{j=2\} \sigma(r)^{2}, \\
\text { with } \kappa_{j, Y_{r}}=\Gamma(j+p+1) m(r) s(r)^{j+p+1} .
\end{gathered}
$$

This is the starting point of cumulant matching between $X_{r}$ and $T_{r}$. In the next result, we allow $r=\infty$, so it applies to any i.d. random variable with finite fourth cumulant.

Proposition 2 (Fourth-order cumulant matching). Fix $0<r \leq \infty$. If $r=\infty$, also assume $\kappa_{4, X_{r}}<\infty$. Then for all large $p$,

$$
\frac{p+4}{p+3}<\frac{\kappa_{2, X_{r}} \kappa_{4, X_{r}}}{\kappa_{3, X_{r}}^{2}} .
$$


For any $p \geq-1$ satisfying $(6)$, if

$$
s(r)=\frac{\kappa_{4, X_{r}}}{(p+4) \kappa_{3, X_{r}}}, \quad m(r)=\frac{\kappa_{3, X_{r}}}{\Gamma(p+4) s(r)^{p+4}},
$$

and if $Y_{r}$ is defined by (3), then $\kappa_{2, X_{r}}>\kappa_{2, Y_{r}}$, and by setting

$$
\sigma(r)=\left(\kappa_{2, X_{r}}-\kappa_{2, Y_{r}}\right)^{1 / 2},
$$

$\kappa_{j, X_{r}}=\kappa_{j, T_{r}}$ for $2 \leq j \leq 4$

Proof. By assumption, $\kappa_{i, X_{r}}<\infty$ for $2 \leq i \leq 4$. By Hölder inequality, $\kappa_{3, X_{r}}^{2}<\kappa_{2, X_{r}} \kappa_{4, X_{r}}$, which implies (6). From (5), by setting $s(r)$ and $m(r)$ as in (7), $\kappa_{j, X_{r}}=\kappa_{j, Y_{r}}$ for $j=3,4$ and

$$
\kappa_{2, Y_{r}}=\Gamma(p+3) m(r) s(r)^{p+3}=\Gamma(p+3) \frac{\kappa_{3, X_{r}}}{\Gamma(p+4) s(r)}=\frac{(p+4) \kappa_{3, X_{r}}^{2}}{(p+3) \kappa_{4, X_{r}}} .
$$

Then for $p \geq-1$ satisfying (6), $\kappa_{2, Y_{r}}<\kappa_{2, X_{r}}$. The rest of the result is then clear.

Proposition 3 (Fifth-order cumulant matching). Let $\lambda(\mathrm{d} u)=\mathbf{1}\{u>0\} u^{-a-1} \ell(u) \mathrm{d} u$, where $a \in$ $(0,2)$ and $\ell(u)$ is slowly varying at $0+$. Let $p=p(r)$ be defined by the equation

$$
1+\frac{1}{p+4}=\frac{\kappa_{3, X_{r}} \kappa_{5, X_{r}}}{\kappa_{4, X_{r}}^{2}}
$$

Then for all small $r>0, p>-1$ and satisfies (6), and by setting $s(r), m(r)$ and $\sigma(r)$ according to (7) and (8), $\kappa_{j, X_{r}}=\kappa_{j, T_{r}}$ for $2 \leq j \leq 5$.

Proof. Since $\ell$ is slowly varying at $0+$, for $j \geq 3$,

$$
\kappa_{j, X_{r}}=\int_{0}^{r} u^{j-1-a} \ell(u) \mathrm{d} u \sim \frac{r^{j-a} \ell(r)}{j-a},
$$

as $r \rightarrow 0+[4,24]$. As a result,

$$
\frac{1}{p+4}=\frac{\kappa_{3, X_{r}} \kappa_{5, X_{r}}}{\kappa_{4, X_{r}}^{2}}-1 \sim \frac{(4-a)^{2}}{(3-a)(5-a)}-1=\frac{1}{(3-a)(5-a)}, \quad r \rightarrow 0+.
$$

It follows that $p \sim a^{2}-8 a+11>-1$. Thus, for all small $r>0, p>-1$. By Proposition 2, it only remains to show that $p$ satisfies (6) and $\kappa_{5, X_{r}}=\kappa_{5, Y_{r}}$. By (9), as $r \rightarrow 0+$,

$$
\frac{\kappa_{2, X_{r}} \kappa_{4, X_{r}}}{\kappa_{3, X_{r}}^{2}} \sim \frac{(3-a)^{2}}{(2-a)(4-a)}=1+\frac{1}{a^{2}-6 a+8} .
$$

Therefore, with $p>-1$, (6) is equivalent to $p>a^{2}-6 a+5$, which holds for $a \in(0,2)$. Finally, that $\kappa_{5, X_{r}}=\kappa_{5, Y_{r}}$ follows from $\kappa_{3, X_{r}} \kappa_{5, X_{r}} / \kappa_{4, X_{r}}^{2}=(p+5) /(p+4)=\kappa_{3, Y_{r}} \kappa_{5, Y_{r}} / \kappa_{4, Y_{r}}^{2}$.

Now we consider the symmetric case. Suppose $X=X^{(1)}-X^{(2)}$, where $X^{(i)}$ are i.i.d. with Lévy measure $\lambda$ supported in $\mathbb{R}_{+}$. Let $X_{r}=X_{r}^{(1)}-X_{r}^{(2)}$, and approximate it by $T_{r}=T_{r}^{(1)}-T_{r}^{(2)}$, where $T_{r}^{(i)}$ are i.i.d. defined in (4). Since all the odd-ordered cumulants of $X_{r}$ and $T_{r}$ are 0, we only need to match their even-ordered cumulants. The next results states that for the general case, we can match their cumulants up to order 7, and for the i.d. distribution as in Proposition 3, we can match their cumulants up to order 9. 
Proposition 4 (Symmetric case). 1) Fix $r>0$. Then for all large $p$,

$$
\frac{(p+5)(p+6)}{(p+3)(p+4)}<\frac{\kappa_{2, X_{r}} \kappa_{6, X_{r}}}{\kappa_{4, X_{r}}^{2}}
$$

For any $p \geq-1$ satisfying (10), if

$$
s(r)=\sqrt{\frac{\kappa_{6, X_{r}}}{(p+5)(p+6) \kappa_{4, X_{r}}}}, \quad m(r)=\frac{\kappa_{4, X_{r}}}{2 \Gamma(p+5) s(r)^{p+5}},
$$

and if $Y_{r}=Y_{r}^{(1)}-Y_{r}^{(2)}$, where $Y_{r}^{(i)}$ are i.i.d. as defined in (3), then $\kappa_{2, X_{r}}>\kappa_{2, Y_{r}}$, and by setting $\sigma(r)$ as in (8), $\kappa_{j, X_{r}}=\kappa_{j, T_{r}}$ for $2 \leq j \leq 7$.

2) If the Lévy measure $\lambda$ of $X^{(i)}$ is $\mathbf{1}\{u>0\} u^{-a-1} \ell(u) \mathrm{d} u$, where $a \in(0,2)$ and $\ell(u)$ is slowly varying at $0+$, then for all small $r>0$, there is a unique $p=p(r)>0$ satisfying (10) and

$$
\frac{(p+7)(p+8)}{(p+5)(p+6)}=\frac{\kappa_{4, X_{r}} \kappa_{8, X_{r}}}{\kappa_{6, X_{r}}^{2}}
$$

Consequently, for this $p$, by setting $s(r)$ and $m(r)$ according to (11) and $\sigma(r)$ according to (8), $\kappa_{j, X_{r}}=\kappa_{j, T_{r}}$ for $2 \leq j \leq 9$.

Proof. 1) By Hölder inequality, $\kappa_{4, X_{r}}^{2}<\kappa_{2, X_{r}} \kappa_{6, X_{r}}$, so for all large $p$, (10) is satisfied. Since for even-valued $j, \kappa_{j, Y_{r}}=2 \kappa_{j, Y_{r}^{(1)}}=2 \Gamma(j+p+1) m(r) s(r)^{j+p+1}$, it is easy to see $\kappa_{4, X_{r}}=\kappa_{4, Y_{r}}$ and $\kappa_{6, X_{r}}=\kappa_{6, Y_{r}}$. On the other hand, for all odd-valued $j, \kappa_{j, X_{r}}=\kappa_{j, Y_{r}}=0$. Finally, by similar argument for Proposition 2, $\kappa_{2, Y_{r}}<\kappa_{2, X_{r}}$, leading to $\kappa_{j, X_{r}}=\kappa_{j, T_{r}}$ for $2 \leq j \leq 7$.

2) Following the proof of Proposition 3,

$$
\frac{\kappa_{4, X_{r}} \kappa_{8, X_{r}}}{\kappa_{6, X_{r}}^{2}} \sim \frac{(6-a)^{2}}{(4-a)(8-a)}=1+\frac{4}{(4-a)(8-a)}:=h(a), \quad r \rightarrow 0+.
$$

Clearly, $h(a)$ is strictly increasing on $(0,2)$. On the other hand,

$$
g(p):=\frac{(p+7)(p+8)}{(p+5)(p+6)}=\left(1+\frac{2}{p+5}\right)\left(1+\frac{2}{p+6}\right)
$$

is strictly decreasing on $(-1, \infty)$, with $g(0)>h(2)>h(a)>h(0)>1=g(\infty)$. Therefore, there is a unique $p>0$ satisfying (12). We have to show that for this $p=p(r),(10)$ is satisfied for all small $r>0$. By continuity, it suffices to show that for $p>0$,

$$
\frac{(p+7)(p+8)}{(p+5)(p+6)}=\frac{(6-a)^{2}}{(4-a)(8-a)} \Longrightarrow \frac{(p+5)(p+6)}{(p+3)(p+4)}<\frac{(4-a)^{2}}{(2-a)(6-a)}
$$

By calculation, the equality is equivalent to $2 p^{2}=2 p\left(a^{2}-12 a+21\right)+13 a^{2}-156 a+356$, while the inequality is equivalent to $2 p^{2}>2 p\left(a^{2}-8 a+5\right)+9 a^{2}-72 a+84$. Then, by $p>0$ and $0<a<2$, the equality indeed implies the inequality. The rest of the proof then follows the one for 1 ).

Propositions 3 and 4 directly lead to the following result on the truncated stable case. Note that for the non-truncated case, simple exact sampling method is known [14]. Also, for $a \in(0,1)$, the truncated stable distribution can be sampled exactly [9]. 
Corollary 1. Let $\lambda(\mathrm{d} u)=c \mathbf{1}\left\{0<u<r_{0}\right\} u^{-a-1} \mathrm{~d} u$, where $c>0, r_{0} \in(0, \infty)$, and $a \in(0,2)$.

1) Suppose $X$ has Lévy measure $\lambda$. If $p=a^{2}-8 a+11$, then $p>-1$ and for all $0<r \leq r_{0}$, by setting $s(r)$ and $m(r)$ according to $(7), \kappa_{2, X_{r}}>\kappa_{2, Y_{r}}$, and by setting $\sigma(r)$ according to (8), $\kappa_{j, X_{r}}=\kappa_{j, T_{r}}$ for $2 \leq j \leq 5$.

2) Suppose $X=X^{(1)}-X^{(2)}$, with $X^{(i)}$ being i.i.d. with Lévy measure $\lambda$. If $p$ is the unique solution in $(0, \infty)$ to

$$
\frac{(p+7)(p+8)}{(p+5)(p+6)}=\frac{(6-a)^{2}}{(4-a)(8-a)}
$$

then for all $0<r \leq r_{0}$, by setting $s(r), m(r)$ according to (11), $\kappa_{2, X_{r}}>\kappa_{2, Y_{r}}$, and by setting $\sigma(r)$ according to (8), $\kappa_{j, X_{r}}=\kappa_{j, T_{r}}$ for $2 \leq j \leq 9$.

\subsection{Error bound for approximation}

We consider the error of approximation of $X$ by $\Delta_{r}+T_{r}$. Denote constants

$$
\begin{aligned}
& C_{1}=\sin 1=0.841 \ldots, \\
& C_{2}=\inf _{p>0} \frac{1}{\Gamma(p)} \int_{0}^{p} u^{p-1} e^{-u} \mathrm{~d} u=\inf _{p>0} \mathbb{P}\left\{\xi_{p} \leq p\right\}, \quad \xi_{p} \sim \operatorname{Gamma}(p, 1) .
\end{aligned}
$$

Note that $C_{2} \in(0,1)$ because

$$
\frac{1}{\Gamma(p)} \int_{0}^{p} u^{p-1} e^{-u} \mathrm{~d} u \sim \frac{1}{\Gamma(p)} \int_{0}^{p} u^{p-1} \mathrm{~d} u \sim p^{p} \rightarrow 1, \quad p \rightarrow 0+
$$

and by Central Limit Theorem, $\mathbb{P}\left\{\xi_{p}-p \leq 0\right\} \rightarrow 1 / 2$ as $p \rightarrow \infty$.

Observe that for $s(r)$ defined in (7) or (11), $s(r)<r /(p+3)$. The main result of the section is the following.

Theorem 1. Fix $r \in(0, \infty)$. Let $T_{r}$ be defined by (3)-(4) for the asymmetric case, or by $T_{r}^{(1)}-T_{r}^{(2)}$ for the symmetric case, with $T_{r}^{(i)}$ i.i.d. defined by $(3)-(4)$. Suppose $s(r)<r /(p+3)$ and $\sigma(r)>0$. Let

$$
L(t, r)=\frac{t^{2}}{2} \min \left\{C_{1}^{2} \kappa_{2, X_{1 /|t|}}, \sigma(r)^{2}\right\} .
$$

For $j \geq 1$, define $Q_{j}(r) \geq 0$ such that

$$
Q_{j}(r)^{2}=\frac{\Gamma(j+1 / 2)}{2\left(C_{1}^{2} C_{2}\right)^{j+1 / 2}}+\kappa_{2, X_{r}}^{j+1 / 2} \int_{1 / r}^{\infty} t^{2 j} e^{-2 L(t, r)} \mathrm{d} t .
$$

Given $q \geq 5$, suppose $\kappa_{j, X_{r}}=\kappa_{j, T_{r}}$ for $2 \leq j<q$. Then

$$
d_{\mathrm{TV}}\left(X, \Delta_{r}+T_{r}\right) \leq \frac{|\kappa|_{q, X_{r}}+|\kappa|_{q, Y_{r}}}{q ! \kappa_{2, X_{r}}^{q / 2}}\left[q Q_{q-1}(r)+Q_{q}(r)+Q_{q+1}(r)\right] .
$$

Remark.

1. The bound is on $d_{\mathrm{TV}}$ instead of the more commonly used $d_{\mathrm{KS}}[1,26]$. However, we have not been able to derive a Berry-Esseen type of bound of the form $C\left(|\kappa|_{q, X_{r}}+|\kappa|_{q, Y_{r}}\right) / \kappa_{2, X_{r}}^{q / 2}$, with $C$ a universal constant only depending on $q$. It appears that some key ingredients for the proof of the Berry-Esseen bound for normal approximation are still missing for higher order approximations. Also, it is likely that the constants in the bounds are not optimal. 
2. The bound will be proved by combining Fourier analysis, the Lindeberg method, and a device in [1] (cf. the proof of Theorem 25.18 in [31]). Although a bound on $d_{\mathrm{KS}}$ may be established solely based on Fourier analysis [10,26], our proof seems to be more transparent and suitable for generalization to multivariate cases.

In the bound for $d_{\mathrm{TV}}\left(X, \Delta_{r}+T_{r}\right), Q_{j}(r)$ look rather technical. We can use the following result to bound them.

Proposition 5. For $b \in(0,1)$ and $q \geq 3$, there is $M=M(b, q)>0$, such that if

$$
\limsup _{r \rightarrow 0+} \frac{\kappa_{2, Y_{r}}}{\kappa_{2, X_{r}}}<b, \quad \liminf _{r \rightarrow 0+} \frac{\kappa_{2, X_{r}}}{r^{2} \ln (1 / r)}>M,
$$

then for any $2 \leq j \leq q+1$,

$$
Q_{j}(r)^{2}=\frac{\Gamma(j+1 / 2)}{2\left(C_{1}^{2} C_{2}\right)^{j+1 / 2}}+o(1), \quad r \rightarrow 0+.
$$

Since the proof is short, we give it here. By (14), for all small $r>0, \sigma(r)^{2}=\kappa_{2, X_{r}}-\kappa_{2, Y_{r}}>$ $(1-b) \kappa_{2, X_{r}}$. Then, from the increasing monotonicity of $\kappa_{2, X_{r}}$ in $r$, there is a constant $c=c(b)>0$, such that for $t \geq 1 / r, L(t, r) \geq c t^{2} \kappa_{2, X_{1 / t}}$. Consequently, if $M \geq(q+2) / c$, then by (14), for $t \geq 1 / r, L(t, r) \geq M c \ln t \geq(q+2) \ln t$, and hence for all $2 \leq j \leq q+1$,

$$
\int_{1 / r}^{\infty} t^{2 j} e^{-2 L(t, r)} \mathrm{d} t \leq \int_{1 / r}^{\infty} t^{2(q+1)-2 M c} \mathrm{~d} t=o(1), \quad r \rightarrow 0+.
$$

Since $\kappa_{2, X_{r}}=o(1)$ as $r \rightarrow 0+$, the proof is complete.

Example 1. Let $\lambda(\mathrm{d} u)=c \mathbf{1}\left\{0<u<r_{0}\right\} u^{-a-1} \mathrm{~d} u$, where $c>0,0<r_{0}<\infty$, and $a \in(0,2)$. By Corollary 1 , given $r \in\left(0, r_{0}\right)$, if $p=a^{2}-8 a+11$, and $s(r), m(r)$ and $\sigma(r)$ are set according $(7)-$ (8), then $\kappa_{j, X_{r}}=\kappa_{j, T_{r}}$ for $2 \leq j<q=6$. To apply (13), we need to get $\kappa_{2, X_{r}},|\kappa|_{6, X_{r}}=\kappa_{6, X_{r}}$, and $|\kappa|_{6, Y_{r}}=\kappa_{6, Y_{r}}$. For $j \geq 2, \kappa_{j, X_{r}}=c r^{j-a} /(j-a)$. Since

$$
\begin{aligned}
s(r) & =\frac{\kappa_{4, X_{r}}}{(p+4) \kappa_{3, X_{r}}}=\frac{(3-a) r}{(p+4)(4-a)}=\frac{r}{(4-a)(5-a)}, \\
\kappa_{2, Y_{r}} & =\frac{\kappa_{3, Y_{r}}}{(p+3) s(r)}=\frac{\kappa_{3, X_{r}}}{(p+3) s(r)}=\frac{c(4-a)(5-a) r^{2-a}}{(3-a)\left(a^{2}-8 a+14\right)},
\end{aligned}
$$

then $\kappa_{6, Y_{r}}=(6+p) s(r) \kappa_{5, Y_{r}}=(6+p) s(r) \kappa_{5, X_{r}}=c A(a) r^{6-a}$, with

$$
A(a)=\frac{a^{2}-8 a+17}{(4-a)(5-a)^{2}} \text {. }
$$

Therefore, by Theorem 1,

$$
d_{\mathrm{TV}}\left(X, \Delta_{r}+T_{r}\right) \leq \frac{(2-a)^{3}}{c^{2}}\left[\frac{1}{6-a}+A(a)\right] \times \frac{6 Q_{5}(r)+Q_{6}(r)+Q_{7}(r)}{6 !} \times r^{2 a} .
$$

Since $0<\kappa_{2, Y_{r}} / \kappa_{2, X_{r}}<1$ is a constant independent of $r$, and $\lambda$ satisfies Orey's condition $\liminf _{r \rightarrow 0+} \kappa_{2, X_{r}} / r^{2-a}>0$ ([29]; also see [31], Proposition 28.3), the conditions in (14) are satisfied no matter the value of $M$. Then by Proposition $5, d_{\mathrm{TV}}\left(X, \Delta_{r}+T_{r}\right)=O\left(r^{2 a}\right)$. This may be compared to the normal approximation in [1,26], where $d_{\mathrm{KS}}$ between $X$ and its normal approximation is of rate $O\left(r^{a / 2}\right)$ when $X$ is asymmetric.

Furthermore, if $X=X^{(1)}-X^{(2)}$ is symmetric, where $X^{(i)}$ are i.i.d. with Lévy measure $\lambda$, then by similar argument while using 2) of Corollary 1 , it can be seen that we can set $q=10$ and get $d_{\mathrm{TV}}\left(X, \Delta_{r}+T_{r}\right)=O\left(r^{4 a}\right)$, whereas the $d_{\mathrm{KS}}$ between $X$ and its normal approximation in this case is of rate $O\left(r^{a}\right)$ [1]. 
Example 2. Let $\lambda(\mathrm{d} u)=1\{u>0\} u^{-a-1} \exp \left(-u^{b}\right) \mathrm{d} u$, where $a \in(0,2)$ and $b>0$. If we directly evaluate $\int_{0}^{r} u^{j} \lambda(\mathrm{d} u)$ for $j \geq 2$, there is no closed formulas available. The following method avoids the problem. Recall that for any odd positive integer $n, e^{-u} \geq f_{n}(u)$ for $u \geq 0$, where $f_{n}(u)=\sum_{i=0}^{n}(-u)^{i} / i$ !. Let $n \geq 1$ be the smallest odd number greater than $a / b-1$ and $F(u)=1\left\{0<u<r_{0}\right\} f_{n}\left(u^{b}\right)$, where $r_{0}=\sup \left\{r>0: f_{n}(u)>0\right.$ for all $\left.0 \leq u<r^{b}\right\}$. Decompose $\lambda(\mathrm{d} u)=\lambda_{1}(\mathrm{~d} u)+\lambda_{2}(\mathrm{~d} u)$, where $\lambda_{1}(\mathrm{~d} u)=\mathbf{1}\{u>0\} u^{-a-1} F(u) \mathrm{d} u$. Because $u^{-a-1}\left[\exp \left(-u^{b}\right)-\right.$ $F(u)]=O\left(u^{(n+1) b-a-1}\right)$ as $u \rightarrow 0+, \lambda_{2}$ has finite mass, and hence corresponds to a compound Poisson random variable that can be sampled exactly. Since $\lambda_{1}(\mathrm{~d} u)=\mathbf{1}\left\{0<u<r_{0}\right\} u^{-a-1} f_{n}\left(u^{b}\right)$, for $0<r<r_{0}$, it is easy to evaluate $\int_{0}^{r} u^{j} \lambda_{1}(\mathrm{~d} u)$. Then we can apply PGN approximation to $\lambda_{1}$. If $X, X^{\prime}$, and $X^{\prime \prime}$ denote i.d. random variables with Lévy measures $\lambda, \lambda_{1}$, and $\lambda_{2}$, respectively, and $\Delta_{r}$ and $T_{r}$ the i.d. random variables from the approximation, then by Proposition 3, we can get $d_{\mathrm{TV}}\left(X, \Delta_{r}+T_{r}+X^{\prime \prime}\right) \leq d_{\mathrm{TV}}\left(X^{\prime}, \Delta_{r}+T_{r}\right)=O\left(r^{2 a}\right)$.

Example 3. Let $\lambda(\mathrm{d} u)=c \mathbf{1}\{0<u<1\} u^{-1} \ln (1 / u) \mathrm{d} u$. Since

$$
\int_{u<r} u^{2} \lambda(\mathrm{d} u)=c \int_{0}^{r} u \ln (1 / u) \mathrm{d} u=\frac{c r^{2}[2 \ln (1 / r)+1]}{4}
$$

by Proposition 2.1 in [1], normal approximation works in the sense that its error in terms of $d_{\mathrm{KS}}$ tends to 0 as $r \rightarrow 0+$. However, since for $|t| \gg 1$,

$$
L(t, r)=\frac{C_{1}^{2} t^{2}}{2} \int_{u<1 /|t|} u^{2} \lambda(\mathrm{d} u) \sim \frac{c C_{1}^{2} \ln |t|}{2},
$$

condition (31) holds only when $c$ is large enough. Furthermore, even when (31) holds, the bound in (13) decreases to 0 very slowly as $r \rightarrow 0$.

\section{Multivariate Poisson-Gamma-Normal approximation}

\subsection{Radial cumulant matching}

In this section, we assume $X \in \mathbb{R}^{d}$ such that in polar coordinates its Lévy measure is

$$
\lambda(\mathrm{d} u, \mathrm{~d} \theta)=\lambda(\mathrm{d} u \mid \theta) \nu(\mathrm{d} \theta), \quad \theta \in S, u>0,
$$

where $S=\left\{\theta \in \mathbb{R}^{d}:\|\theta\|=1\right\}, \nu$ is a finite measure on $S$, and for each $\theta \in S, \lambda(\mathrm{d} u \mid \theta)$ is a Lévy measure on $(0, \infty)$. For symmetric $X, \lambda(\mathrm{d} u \mid \theta)=\lambda(\mathrm{d} u \mid-\theta)$ and $\nu(\mathrm{d} \theta) \equiv \nu(-\mathrm{d} \theta)$. Without loss of generality, assume

$\operatorname{sppt}(\lambda)$ is bounded and not contained in a linear space of lower dimension.

In particular, the assumption implies $\mathbb{E}\|X\|^{p}<\infty$ for all $p>0$.

The so-called radial cumulant matching is as follows. For each $\theta \in S$, find $\sigma(\theta) \geq 0$ and a Lévy measure $\gamma(\mathrm{d} u \mid \theta)$ on $(0, \infty)$, such that, first, for some $q>2$,

$$
\int u^{j} \lambda(\mathrm{d} u \mid \theta)=\mathbf{1}\{j=2\} \sigma(\theta)^{2}+\int u^{j} \gamma(\mathrm{d} u \mid \theta) \quad \nu \text {-a.e. } \theta
$$

for all $2 \leq j<q$ if $X$ is asymmetric, or for all even valued $j \geq 2$ less than $q$ if $X$ is symmetric, and second, for $\theta \neq \theta^{\prime}$, if $\lambda(\mathrm{d} u \mid \theta)=\lambda\left(\mathrm{d} u \mid \theta^{\prime}\right)$, then $\sigma(\theta)=\sigma\left(\theta^{\prime}\right)$ and $\gamma(\mathrm{d} u \mid \theta)=\gamma\left(\mathrm{d} u \mid \theta^{\prime}\right)$. Let $T$ be an i.d. random variable with

$$
\Psi_{T}(t)=\int\left[\frac{1}{2} \sigma(\theta)^{2}\langle t, \theta\rangle^{2}+\int\left(1+\mathrm{i}\langle t, \theta\rangle u-e^{\mathrm{i}\langle t, \theta\rangle u}\right) \gamma(\mathrm{d} u \mid \theta)\right] \nu(\mathrm{d} \theta) .
$$


Then $\mathbb{E} T=0$. If $X$ is asymmetric, then for any $\alpha$ with $1<|\alpha|<q$,

$$
\kappa_{\alpha, T}=\int \theta^{\alpha}\left[\mathbf{1}\{|\alpha|=2\} \sigma(\theta)^{2}+\int u^{|\alpha|} \gamma(\mathrm{d} u \mid \theta)\right] \nu(\mathrm{d} \theta)=\int \theta^{\alpha} u^{|\alpha|} \lambda(\mathrm{d} u \mid \theta) \nu(\mathrm{d} \theta)
$$

which is just $\kappa_{\alpha, X}$. If $X$ is symmetric, the equality holds for any $\alpha$ with $|\alpha|$ being even and $1<|\alpha|<q$. On the other hand, if $|\alpha|$ is odd, then $\kappa_{\alpha, X}=0$ and from the construction of $\gamma$, $\kappa_{\alpha, T}=0$. Therefore, $X$ and $T$ have the same cumulants up to order $q-1$.

The Gaussian component of $T$ has covariance $\int \theta \theta^{\prime} \sigma(\theta)^{2} \nu(\mathrm{d} \theta)$, which can be difficult to evaluate. For normal approximation, the issue can be circumvented by using the asymptotic of the covariance [11]. However, this approach rules out higher order approximation. We propose the following solution. Since $\lambda(\mathrm{d} u \mid \theta)$ is a Lévy measure on $(0, \infty)$ for each $\theta \in S$, given $\tau>0$, it is possible to select $r=r(\theta)>0$ and then set $p=p(\theta), m(r)=m(r, \theta)$, and $s(r)=s(r, \theta)$ as in Propositions 2, 3 , or 4 , such that, letting $\gamma(\mathrm{d} u \mid \theta)=m(r) \mathbf{1}\{u>0\} u^{p} e^{-u / s(r)} \mathrm{d} u$,

$$
\sigma(\theta)^{2}=\int \mathbf{1}\{u<r\} u^{2} \lambda(\mathrm{d} u \mid \theta)-\int u^{2} \gamma(\mathrm{d} u \mid \theta)=\tau^{2}>0, \quad \nu \text {-a.e. } \theta \in S \text {. }
$$

With this choice of $\gamma(\mathrm{d} u \mid \theta)$, the Gaussian component is $N\left(0, \tau^{2} K_{\nu}\right)$, where

$$
K_{\nu}=\int \theta \theta^{\prime} \nu(\mathrm{d} \theta)
$$

can be much more manageable. This is the same matrix identified in formula (3.17) of [11]. By the assumption on $\lambda, K_{\nu}$ is positive definite (p.d.).

There is some flexibility in choosing $\nu$. Given $w(\theta)$ measurable on $S$ with $0<\operatorname{ess} \inf w \leq$ ess $\sup w<\infty$ under $\nu, \lambda(\mathrm{d} u, \mathrm{~d} \theta)$ can be written as $\tilde{\lambda}(\mathrm{d} u \mid \theta) \tilde{\nu}(\mathrm{d} \theta)$, where

$$
\tilde{\lambda}(\mathrm{d} u \mid \theta)=w(\theta) \lambda(\mathrm{d} u \mid \theta), \quad \tilde{\nu}(\mathrm{d} \theta)=\nu(\mathrm{d} \theta) / w(\theta) .
$$

If $r, p, m(r)$ and $s(r)$ are set according to $\tilde{\lambda}(\mathrm{d} u \mid \theta)$ instead of $\lambda(\mathrm{d} u \mid \theta)$, then the matrix in (16) becomes $\int \theta \theta^{\prime} \tilde{\nu}(\mathrm{d} \theta)$. This allows one to choose $w(\theta)$ to simplify the evaluation of the matrix.

In this setting, $\tau$ instead of $r$ is the parameter, and $r, p, m(r)$, and $s(r)$ are functions of $(\tau, \theta)$. We denote the functions by $r_{\tau}(\theta), p_{\tau}(\theta), m_{\tau}(\theta)$ and $s_{\tau}(\theta)$, respectively. Evidently,

$$
\int \mathbf{1}\left\{u<r_{\tau}(\theta)\right\} u^{2} \lambda(\mathrm{d} u \mid \theta)-\int_{0}^{\infty} m_{\tau}(\theta) u^{p_{\tau}(\theta)+2} e^{-u / s_{\tau}(\theta)} \mathrm{d} u=\tau^{2} \quad \nu \text {-a.e. } \theta \in S .
$$

Additionally,

$$
\lambda(\mathrm{d} u \mid \theta)=\lambda\left(\mathrm{d} u \mid \theta^{\prime}\right) \Longrightarrow f(\theta)=f\left(\theta^{\prime}\right), \text { for } f=r_{\tau}, p_{\tau}, m_{\tau}, s_{\tau} \text { for all } \tau>0 .
$$

Now define Lévy measures

$$
\begin{aligned}
& \lambda_{\tau}(\mathrm{d} u, \mathrm{~d} \theta)=\lambda_{\tau}(\mathrm{d} u \mid \theta) \nu(\mathrm{d} \theta), \text { with } \lambda_{\tau}(\mathrm{d} u \mid \theta)=\mathbf{1}\left\{u<r_{\tau}(\theta)\right\} \lambda(\mathrm{d} u \mid \theta) \nu(\mathrm{d} \theta), \\
& \gamma_{\tau}(\mathrm{d} u, \mathrm{~d} \theta)=\gamma_{\tau}(\mathrm{d} u \mid \theta) \nu(\mathrm{d} \theta), \text { with } \gamma_{\tau}(\mathrm{d} u \mid \theta)=m_{\tau}(\theta) \mathbf{1}\{u>0\} u^{p_{\tau}(\theta)} e^{-u / s_{\tau}(\theta)} \mathrm{d} u .
\end{aligned}
$$

Then, for suitable $q \geq 5$, which depends on how $r_{\tau}, p_{\tau}, m_{\tau}$, and $s_{\tau}$ are constructed,

$$
\int u^{j} \lambda_{\tau}(\mathrm{d} u \mid \theta)=\mathbf{1}\{j=2\} \tau^{2}+\int u^{j} \gamma_{\tau}(\mathrm{d} u \mid \theta), \quad \nu \text {-a.s. } \theta \in S
$$


for all $2 \leq j<q$ if $X$ is asymmetric, or for all even valued $j \geq 2$ less than $q$ if $X$ is symmetric. Next, decompose $X$ as the sum of independent i.d. random variables $X_{\tau}$ and $\Delta_{\tau}$, with

$$
\Psi_{X_{\tau}}(t)=\int\left(1+\mathrm{i} u\langle t, \theta\rangle-e^{\mathrm{i} u\langle t, \theta\rangle}\right) \lambda_{\tau}(\mathrm{d} u, \mathrm{~d} \theta),
$$

and $\Psi_{\Delta_{\tau}}(t)=\Psi_{X}(t)-\Psi_{X_{\tau}}(t)$. Then approximate $X_{\tau}$ by

$$
T_{\tau}=Y_{\tau}+\tau Z, \quad \text { with } Z \sim N\left(0, K_{\nu}\right) \text { independent of } Y_{r},
$$

where $Y_{\tau}$ is i.d. with mean 0 and no Gaussian component, and with Lévy measure $\gamma_{\tau}$. Finally, $X$ is approximated by $\Delta_{\tau}+T_{\tau}$.

Clearly, in order for the solution to be valid, $\lambda_{\tau}$ and $\gamma_{\tau}$ have to be valid Lévy measures. First, this means $\mathbf{1}\left\{u<r_{\tau}(\theta)\right\}$ and $m_{\tau}(\theta) \mathbf{1}\{u>0\} u^{p_{\tau}(\theta)} e^{-u / s_{\tau}(\theta)}$ must be measurable functions of $(u, \theta)$. In many cases, the measurability is not difficult to verify. Provided it is established, $\lambda_{\tau}$ immediately is a valid Lévy measure. On the other hand, since $\int u^{2} \gamma_{\tau}(\mathrm{d} u, \mathrm{~d} \theta) \leq \int u^{2} \lambda(\mathrm{d} u, \mathrm{~d} \theta)<\infty$, by the comments in Section 2.2, $\gamma_{\tau}$ is a valid Lévy measure and $\mathbb{E}\left\|Y_{\tau}\right\|^{2}<\infty$.

The solution shifts the burden of evaluating the normal covariance to the sampling of $\Delta_{\tau}$ and $Y_{\tau}$. For the latter, the following statements are true.

Proposition 6. 1) If

$$
\int_{S} B_{\tau}(\theta) \nu(\mathrm{d} \theta)<\infty \quad \text { with } B_{\tau}(\theta)=\int 1\left\{u \geq r_{\tau}(\theta)\right\} \lambda(\mathrm{d} u \mid \theta),
$$

then $\Delta_{\tau} \sim \tilde{\zeta}_{1} \tilde{\omega}_{1}+\cdots+\tilde{\zeta}_{N} \tilde{\omega}_{N}-\tilde{\mu}$, where $\left\{\tilde{\omega}_{i}\right\}$ is a Poisson process on $S$ with Lévy measure $B_{\tau} \mathrm{d} \nu$, conditional on $\left\{\tilde{\omega}_{i}\right\}, \tilde{\zeta}_{1}, \ldots, \tilde{\zeta}_{N}$ are independent, with $\tilde{\zeta}_{i} \sim \mathbf{1}\left\{u \geq r_{\tau}\left(\tilde{\omega}_{i}\right)\right\} \lambda\left(\mathrm{d} u \mid \tilde{\omega}_{i}\right) / B_{\tau}\left(\tilde{\omega}_{i}\right)$, and

$$
\tilde{\mu}=\int \theta u \mathbf{1}\left\{u \geq r_{\tau}(\theta)\right\} \lambda(\mathrm{d} u \mid \theta) \nu(\mathrm{d} \theta) .
$$

2) If $p_{\tau}(\theta)>-1$ for $\nu$-a.e. $\theta$ and

$$
\int_{S} N_{\tau}(\theta) \nu(\mathrm{d} \theta)<\infty \quad \text { with } N_{\tau}(\theta)=\Gamma\left(p_{\tau}(\theta)+1\right) m_{\tau}(\theta) s_{\tau}(\theta)^{p_{\tau}(\theta)+1}
$$

then $Y_{\tau} \sim \zeta_{1} \omega_{1}+\cdots+\zeta_{N} \omega_{N}-\mu$, where $\left\{\omega_{i}\right\}$ is a Poisson process on $S$ with Lévy measure $N_{\tau} \mathrm{d} \nu$, conditional on $\left\{\omega_{i}\right\}, \zeta_{1}, \ldots, \zeta_{N}$ are independent, with $\zeta_{i} \sim \operatorname{Gamma}\left(p_{\tau}\left(\omega_{i}\right), s_{\tau}\left(\omega_{i}\right)\right)$, and

$$
\mu=\int_{S} \theta\left(p_{\tau}(\theta)+1\right) s_{\tau}(\theta) N_{\tau}(\theta) \nu(\mathrm{d} \theta)
$$

3) If $X$ is symmetric, then $\tilde{\mu}=\mu=0$.

4) If $\lambda$ is direction independent, i.e, $\lambda(\mathrm{d} u \mid \theta)=\lambda_{0}(\mathrm{~d} u)$ for $\nu$-a.e. $\theta \in S$ for some Lévy measure $\lambda_{0}$, then given $\tau>0, r_{\tau}, m_{\tau}, p_{\tau}, s_{\tau}$, and $N_{\tau}$ are $\nu$-a.e. constant, and $\tilde{\mu}=\theta_{\nu} \int u \mathbf{1}\left\{u \geq r_{\tau}\right\} \lambda_{0}(\mathrm{~d} u)$, $\mu=\theta_{\nu}\left(p_{\tau}+1\right) s_{\tau} N_{\tau}$, where $\theta_{\nu}=\int_{S} \theta \nu(\mathrm{d} \theta)$.

Proof. 1) It is easy to see $\Delta_{\tau}$ has mean 0 and no Gaussian component, and its Lévy measure is $1\left\{u>r_{\tau}(\theta)\right\} \lambda(\mathrm{d} u \mid \theta) \nu(\mathrm{d} \theta)$, which by assumption (20) has a finite mass. Then 1) follows from standard results on compound Poisson processes [12]. The proof of 2) is similar. Finally, both 3) and 4) follow from the construction of the functions $r_{\tau}(\theta), p_{\tau}(\theta), s_{\tau}(\theta)$, and $m_{\tau}(\theta)$. 
To apply the result, it is desirable that

$$
\operatorname{ess} \sup B_{\tau}(\theta)<\infty, \quad \operatorname{ess} \sup N_{\tau}(\theta)<\infty \quad \text { under } \nu,
$$

because $\left\{\tilde{\omega}_{i}\right\}$ and $\left\{\omega_{i}\right\}$ then can be sampled using the standard thinning method ([14], p. 253-255), as long as it is easy to sample the Poisson process on $S$ with Lévy measure $C \nu$ for any $C>0$. In this case, there is no need to know all $B_{\tau}(\theta)$ and $N_{\tau}(\theta)$ beforehand. Parts 3) and 4) of Proposition 6 lists two cases where $\tilde{\mu}$ and $\mu$ are calculable. It follows that the quantities are still calculable if $\lambda$ is the sum of a symmetric Lévy measure and a Lévy measure $\lambda^{\prime}$ that is "piecewise" direction independent, i.e., $\lambda^{\prime}(\mathrm{d} u \mid \theta)=\lambda_{i}(\mathrm{~d} u)$ for $\nu$-a.s. $\theta \in S_{i}, i=1, \ldots, n$, where $S_{i}$ form a partition of $S$. However, in other cases, $\tilde{\mu}$ and $\mu$ may not have closed form expressions. This is the limitation alluded in Introduction.

Example 4. Let $\lambda(\mathrm{d} u, \mathrm{~d} \theta)=\mathbf{1}\left\{0<u<r_{0}\right\} u^{-a(\theta)-1} \mathrm{~d} u \nu(\mathrm{d} \theta)$, where $r_{0} \in(0, \infty)$ and $a(\theta) \in(0,2)$ is a measurable function on $S$, such that

$$
0<a_{0}:=\operatorname{essinf} a \leq \operatorname{ess} \sup a=a_{1}<2 \text { under } \nu \text {. }
$$

Suppose also that $\lambda$ is symmetric. For $a \in(0,2)$, let $\pi(a)$ be the unique solution in $(0, \infty)$ to

$$
\frac{(p+7)(p+8)}{(p+5)(p+6)}=\frac{(6-a)^{2}}{(4-a)(8-a)}
$$

Since $\lambda(\mathrm{d} u \mid \theta)=\mathbf{1}\left\{0<u<r_{0}\right\} u^{-a(\theta)-1} \mathrm{~d} u$, in light of Corollary 1 , define $r_{\tau}(\theta), p_{\tau}(\theta), s_{\tau}(\theta)$, and $m_{\tau}(\theta)$ as follows. First, let $p_{\tau}(\theta)=\pi(a(\theta))$. Next, provided $r_{\tau}(\theta) \in\left(0, r_{0}\right)$, define

$$
s_{\tau}(\theta)=J_{1}(a(\theta)) r_{\tau}(\theta), \quad m_{\tau}(\theta)=J_{2}(a(\theta)) r_{\tau}(\theta)^{-1-a(\theta)-\pi(a(\theta))}
$$

according to (11), where

$$
J_{1}(a)=\sqrt{\frac{(4-a)}{(\pi(a)+5)(\pi(a)+6)(6-a)}}, \quad J_{2}(a)=\frac{1}{2 \Gamma(\pi(a)+5)(4-a) J_{1}(a)^{\pi(a)+5}}
$$

are strictly positive and continuous on $(0,2)$. This yields $\int u^{2} \lambda_{\tau}(\mathrm{d} u \mid \theta)=J_{0}(a(\theta)) r_{\tau}(\theta)^{2-a(\theta)}$, where $J_{0}(a)=1 /(2-a)$, and

$$
\int u^{2} \gamma_{\tau}(\mathrm{d} u \mid \theta)=\Gamma(\pi(a(\theta))+3) m_{\tau}(\theta) s(r)^{\pi(a(\theta))+3}=J_{3}(a(\theta)) r_{\tau}(\theta)^{2-a(\theta)}
$$

where $J_{3} \in C(0,2)$ is strictly positive. In particular, $0<J_{3}(a)<J_{0}(a)$. Finally, from condition (17), it follows that if $r_{\tau}(\theta) \in\left(0, r_{0}\right)$, then $r_{\tau}(\theta)=J_{4}(a(\theta)) \tau^{2 /(2-a(\theta))}$, where

$$
J_{4}(a)=\left[J_{0}(a)-J_{3}(a)\right]^{-1 /(2-a)} \in C(0,2) .
$$

By assumption (22), for all small $\tau>0$, ess sup $J_{4}(a(\theta)) \tau^{2 /(2-a(\theta))}<r_{0}$, and hence all the above definitions are valid. Since $\pi(a)$ and all $J_{i}(a)$ are continuous functions, $\pi(a(\theta))$ and $J_{i}(a(\theta))$ are measurable functions of $\theta$. It is then easy to see $\mathbf{1}\left\{u<r_{\tau}(\theta)\right\}$ is a measurable function of $(u, \theta)$ and hence $\lambda_{\tau}$ is a valid Lévy measure. Likewise, $\gamma_{\tau}$ is a valid Lévy measure. Consequently, by Corollary 1 and the symmetry of $\lambda$, (19) is satisfied with $q=10$. 
We consider the sampling of $\Delta_{\tau}$ and $Y_{\tau}$ based on Proposition 6. Given $\tau>0$ small enough, by $r_{\tau}(\theta)=J_{4}(a(\theta)) \tau^{2 /(2-a(\theta))}$ and $(22)$,

$$
\operatorname{ess} \sup B(\theta)=\operatorname{ess} \sup \int_{r_{\tau}(\theta)}^{r_{0}} u^{-a(\theta)-1} \mathrm{~d} u \leq \operatorname{ess} \sup \frac{r_{\tau}(\theta)^{-a(\theta)}}{a(\theta)}<\infty .
$$

Since $\lambda$ is symmetric, then by Proposition $6, \Delta_{\tau}=\tilde{\zeta}_{1} \tilde{\omega}_{1}+\cdots+\tilde{\zeta}_{N} \tilde{\omega}_{N}$, where $\left\{\tilde{\omega}_{i}\right\}$ is a Poisson process that can be sampled using the thinning method, while conditioning on $\tilde{\omega}_{i}$, the probability density of $\tilde{\zeta}_{i}$ is proportional to $\mathbf{1}\left\{r_{\tau}\left(\tilde{\omega}_{i}\right) \leq u<r_{0}\right\} u^{-a\left(\tilde{\omega}_{i}\right)-1}$. On the other hand, by the construction of $p_{\tau}, m_{\tau}$ and $s_{\tau}, N_{\tau}(\theta)=J(a(\theta)) r_{\tau}(\theta)^{-a(\theta)}$ for some continuous $J(a)>0$, so again by (22), $\operatorname{ess} \sup N(\theta)<\infty$. Then $Y_{\tau}=\zeta_{1} \omega_{1}+\cdots+\zeta_{N} \omega_{N}$, where $\left\{\omega_{i}\right\}$ is a Poisson process that can be sampled using the thinning method, while conditioning on $\omega_{i}, \zeta_{i} \sim \operatorname{Gamma}\left(\pi\left(a\left(\omega_{i}\right)\right), s_{\tau}\left(\omega_{i}\right)\right)$.

Unfortunately, unlike the univariate case, currently there are no computationally efficient methods to sample $\Delta_{\tau}$ or $Y_{\tau}$, other than sampling individual $\left(\tilde{\omega}_{i}, \tilde{\zeta}_{i}\right)$ or $\left(\omega_{i}, \zeta_{i}\right)$ and then taking the sum of $\tilde{\zeta}_{i} \tilde{\omega}_{i}$ or $\zeta_{i} \omega_{i}$. This raises the issue of computational complexity of the PGN approximation. In the next subsection, after obtaining an error bound for the PGN approximation, we will come back to the issue and argue that, in some cases, comparing to normal approximation [11], the improvement in error rate is worth the extra computation complexity, at least asymptotically.

Finally, we remark that if $\nu(\mathrm{d} \theta)=w(\theta) \sigma(\mathrm{d} \theta)$, where $\sigma$ is the spherical measure on $S$ and $w(\theta)$ is measurable on $S$ with $0<\operatorname{essinf} w \leq \operatorname{ess} \sup w<\infty$ under $\sigma$, then by setting $r_{\tau}(\theta)=$ $J_{4}(a(\theta))\left[\tau^{2} / w(\theta)\right]^{1 /(2-a(\theta))}$ and adjusting $s_{\tau}(\theta)$ and $m_{\tau}(\theta)$ accordingly, we get $K_{\nu}=\int \theta \theta^{\prime} \sigma(\mathrm{d} \theta)=$ $I / d$. With $\sigma(\mathrm{d} \theta)$ being the new $\nu(\mathrm{d} \theta)$, the sampling of $\Delta_{\tau}$ and $Y_{\tau}$ can be done as above.

Example 5. Normal approximation of tempered stable processes is studied in detail in [11]. For such a process, $\lambda(\mathrm{d} u \mid \theta)=\mathbf{1}\{u>0\} u^{-a-1} h(u, \theta) \mathrm{d} u$, where for each $\theta, h(u, \theta)$ is a completely monotone function with $h(0+, \theta)=1$ and $h(\infty, \theta)=0$. A generalized shot noise series representation is used in [11] for normal approximation. Although the resulting covariance of the normal distribution in general has no closed form, it is shown that by using its asymptotic, the normal distribution still works.

In the context of PGN approximation to the i.d. distribution with Lévy measure $\lambda$, we have to evaluate the covariance precisely. At this point, a solution to the general case has not been found. However, suppose $h(u, \theta)$ can be written as

$$
h(u, \theta)=1-h_{1}(\theta) u+h_{2}(u, \theta) u^{2},
$$

such that $0 \leq \operatorname{ess} \inf h_{1} \leq \operatorname{ess} \sup h_{1}<\infty$ under $\nu$ and $0 \leq \operatorname{essinf} h_{2} \leq \operatorname{ess} \sup h_{2}<\infty$ under $\ell \times \nu$, where $\ell$ is the Lebesgue measure, then the following method can be used. First, fix $r_{0}$ such that $1-h_{1}(\theta) r_{0} \geq 0$ for $\nu$-a.e. $\theta$. Let

$$
\mu(\mathrm{d} u \mid \theta)=\mathbf{1}\left\{0<u<r_{0}\right\} u^{-a-1}\left[1-h_{1}(\theta) u\right] \mathrm{d} u
$$

and $\mu(\mathrm{d} u, \mathrm{~d} \theta)=\mu(\mathrm{d} u \mid \theta) \nu(\mathrm{d} \theta)$. Decompose $\lambda=\mu+\lambda_{1}$, with $\lambda_{1}=\lambda-\mu$. For $0<u<r_{0}$, $\lambda_{1}(\mathrm{~d} u \mid \theta)=u^{-a-1}\left[h(u, \theta)-1+h_{1}(\theta) u\right] \mathrm{d} u \nu(\mathrm{d} \theta)=u^{-a+1} h_{2}(\theta, u) \mathrm{d} u \nu(\mathrm{d} \theta)$, while for $u \geq r_{0}$, $\lambda_{1}(\mathrm{~d} u \mid \theta)=\lambda(\mathrm{d} u \mid \theta)$. As a result, $\lambda_{1}$ is a Lévy measure with

$$
\int \lambda_{1}(\mathrm{~d} u, \mathrm{~d} \theta)=\int \nu(\mathrm{d} \theta) \int_{0}^{r_{0}} u^{-a+1} h_{2}(\theta, u) \mathrm{d} u+\int \mathbf{1}\left\{u \geq r_{0}\right\} \lambda(\mathrm{d} u, \mathrm{~d} \theta)<\infty
$$

and hence it gives rise to a compound Poisson random variable. We therefore only need to apply PGN approximation to $\mu$. It is easy to compute $\int u^{j} \mu(\mathrm{d} u \mid \theta)$ for $j \geq 2$. Then the functions $r_{\tau}(\theta)$, $p_{\tau}(\theta), s_{\tau}(\theta)$, and $m_{\tau}(\theta)$ can be fixed following Example 4, although the calculation is more tedious due to the extra term $h_{1}(\theta) u$. 


\subsection{Error bound for approximation}

We next consider how well $X$ is approximated by $\Delta_{\tau}+T_{\tau}$. For any symmetric p.d. matrix $\Sigma$, denote by $\Sigma^{1 / 2}$ the unique symmetric p.d. matrix whose square is equal to $\Sigma$. Given $\tau>0$, let

$$
\Sigma_{\tau}=\mathbb{V}\left(X_{\tau}\right)=\mathbb{V}\left(T_{\tau}\right), \quad A_{\tau}=\Sigma_{\tau}^{1 / 2}, \quad K^{*}=\tau^{2} A_{\tau}^{-1} K_{\nu} A_{\tau}^{-1} .
$$

Let $C_{1}$ and $C_{2}$ be the same constants defined in Section 3.2. Denote, for $z>0$,

$$
\begin{gathered}
\varrho_{\tau}(z)=\text { smallest eigenvalue of } C_{1}^{2} A_{\tau}^{-1} M_{\tau}(z) A_{\tau}^{-1} \text { and } K^{*} \\
\text { with } M_{\tau}(z)=\int u^{2} \theta \theta^{\prime} \mathbf{1}\left\{u<r_{\tau}(\theta) \wedge \frac{z}{\left\|A_{\tau}^{-1} \theta\right\|}\right\} \lambda_{\tau}(\mathrm{d} u \mid \theta) \nu(\mathrm{d} \theta) .
\end{gathered}
$$

Finally, denote

$$
h(d)=\lfloor d / 2\rfloor+1 .
$$

Theorem 2. Given $\tau>0$, suppose that under $\nu$,

$$
\begin{gathered}
\operatorname{essinf} p_{\tau}(\theta) \geq-1, \quad \operatorname{ess} \sup \frac{\left[p_{\tau}(\theta)+3\right] s_{\tau}(\theta)}{r_{\tau}(\theta)} \leq 1 \\
R^{*}:=\operatorname{ess} \sup \left\|A_{\tau}^{-1} \theta\right\| r_{\tau}(\theta) \leq 1, \quad S^{*}:=\operatorname{ess} \sup \left[p_{\tau}(\theta)+h(d)+1\right]\left\|A_{\tau}^{-1} \theta\right\| s_{\tau}(\theta) \leq 1
\end{gathered}
$$

and for some $q \geq 5, \kappa_{\alpha, X_{\tau}}=\kappa_{\alpha, T_{\tau}}$ for $2 \leq|\alpha|<q$ and

$$
\int_{0}^{\infty} s^{2 q+2 h(d)+d-1} e^{-\varrho_{\tau}(1 / s) s^{2}} \mathrm{~d} s<\infty .
$$

Then

$$
d_{\mathrm{TV}}\left(X, \Delta_{\tau}+T_{\tau}\right) \leq \frac{G(d, q, \tau)}{q !}\left[\int\left\|u A_{\tau}^{-1} \theta\right\|_{1}^{q} \lambda_{\tau}(\mathrm{d} u, \mathrm{~d} \theta)+\int\left\|u A_{\tau}^{-1} \theta\right\|_{1}^{q} \gamma_{\tau}(\mathrm{d} u, \mathrm{~d} \theta)\right]
$$

where $\|x\|_{1}$ stands for the $L^{1}$ norm $\left|x_{1}\right|+\cdots+\left|x_{d}\right|$ and

$$
G(d, q, \tau)=c(d, q) \sqrt{1+\int_{1 / R^{*}}^{\infty} L_{d, q}(s) e^{-\varrho_{\tau}(1 / s) s^{2}} \mathrm{~d} s}
$$

with $c(d, q)$ being a constant only depending on $(d, q)$ and $L_{d, q}(s)$ a polynomial of order no greater than $2 q+2 h(d)+d-1$ whose coefficients are constants only depending on $(d, q)$.

Remark.

1. Although $A_{\tau}$ appears in the bound, it is not used in the actual construction of $T_{\tau}$ or $\Delta_{\tau}$, and therefore does not generate a computational problem.

2. A drawback of the bound in Theorem 2 is that, although asymptotically, the error rate can be significantly better than normal approximation, the constant coefficients in the bound, i.e., $c(d, q)$ and those in $L_{d, q}(s)$, are very large even for modest $d$. Perhaps alternative methods for normal approximation (e.g. [3, 8, 28]) could be employed to improve these terms, or even replace $G(d, q, \tau)$ with a universal constant that only depends on $(d, q)$.

In Theorem 2, the inequalities in (24) are the easiest to establish. On the other hand, $R^{*}$ and $S^{*}$ need more careful treatment as they involve $A_{\tau}$. By $(24), S^{*}$ may be bounded via $R^{*}$. The main technical term in Theorem 2 is $G(d, q, \tau)$. The next result, which will be proved in Section 5.2 , provides some simple criteria to bound $R^{*}$ and $G(d, q, \tau)$. 
Proposition 7. Under $\nu$, the following statements are true.

1) Let $c=c\left(K_{\nu}\right)>0$ be a square root of the smallest eigenvalue of $K_{\nu}$. Then $\sup _{S}\left\|A_{\tau}^{-1} \theta\right\| \leq$ $1 /(c \tau)$. If

$$
\lim _{\tau \rightarrow 0+} \operatorname{ess} \sup r_{\tau}(\theta) / \tau=0
$$

then $R^{*}=o(1)$ as $\tau \rightarrow 0$.

2) Given $b>1$ and $q \geq 5$, there is $M=M\left(b, q, K_{\nu}\right)>0$, such that, if, in addition to (28),

$$
\begin{gathered}
\limsup _{\tau \rightarrow 0+} \frac{1}{\tau^{2}} \operatorname{ess} \sup \int u^{2} \lambda_{\tau}(\mathrm{d} u \mid \theta)<b, \\
\liminf _{r \rightarrow 0+} \frac{1}{r^{2} \ln (1 / r)} \operatorname{essinf} \int u^{2} \mathbf{1}\{u<r\} \lambda(\mathrm{d} u \mid \theta)>M,
\end{gathered}
$$

then (26) holds and $G(d, q, \tau)=c(d, q)+o(1)$ as $\tau \rightarrow 0$. (Note the variate in (30) is $r$, not $\tau$.)

Example 5 (Continued). Note the assumption in (22). By $r_{\tau}(\theta)=J_{4}(a(\theta)) \tau^{2 /(2-a(\theta))},(28)$ is satisfied. By $\int u^{2} \lambda_{\tau}(\mathrm{d} u \mid \theta)=J_{0}(a(\theta)) r_{\tau}(\theta)^{2-a(\theta)}=J(a(\theta)) \tau^{2}$ for some $J(a) \in C(0,2)$, (29) is satisfied. Since $\int u^{2} \mathbf{1}\{u<r\} \lambda(\mathrm{d} u \mid \theta)=r^{2-a(\theta)} /(2-a(\theta))$, then (30) is satisfied no matter the value of $M$. Thus we can apply Proposition 7. Since $p_{\tau}(\theta)=\pi(a(\theta))>0$ and $\left(p_{\tau}(\theta)+\right.$ 3) $s_{\tau}(\theta) / r_{\tau}(\theta)=\left(p_{\tau}(\theta)+3\right) J_{1}(a)<1$, the conditions in (24) are satisfied. The last condition we need to check that for small $\tau>0, S^{*} \leq 1$ in (25). However, by (24) and ess $\sup p_{\tau}(\theta)=$ ess $\sup \pi(a(\theta))<\infty, S^{*}=O\left(R^{*}\right)=o(1)$.

We now can apply Theorem 2. By $\|x\|_{1} \leq \sqrt{d}\|x\|$ for $x \in \mathbb{R}^{d}$ and by $\left\|A_{\tau}^{-1} \theta\right\| \leq 1 /(c \tau)$, where $c$ is the constant in Proposition 7,

$$
\begin{aligned}
\int\left\|u A_{\tau}^{-1} \theta\right\|_{1}^{q} \lambda_{\tau}(\mathrm{d} u, \mathrm{~d} \theta) & \leq d^{q / 2} \int u^{q}\left\|A_{\tau}^{-1} \theta\right\|_{2}^{q} \lambda_{\tau}(\mathrm{d} u, \mathrm{~d} \theta) \\
& \leq d^{q / 2}(c \tau)^{-q} \int u^{q} \lambda_{\tau}(\mathrm{d} u, \mathrm{~d} \theta) \\
& =(c \tau / \sqrt{d})^{-q} \int \frac{r_{\tau}(\theta)^{q-a(\theta)}}{q-a(\theta)} \nu(\mathrm{d} \theta) \leq c^{\prime} \tau^{(q-2) a_{0} /\left(2-a_{0}\right)},
\end{aligned}
$$

where $c^{\prime}$ is a constant independent of $\tau$. For $\int\left\|u A_{\tau}^{-1} \theta\right\|_{1}^{q} \gamma_{\tau}(\mathrm{d} u, \mathrm{~d} \theta)$, a similar bound holds. Combining these bounds and Proposition $7, d_{\mathrm{TV}}\left(X, \Delta_{\tau}+T_{\tau}\right)=O\left(\tau^{(q-2) a_{0} /\left(2-a_{0}\right)}\right)$, where $q=10$.

Finally, we compare the computational complexity of the above PGN approximation and the normal approximation for $X$ [11]. To make a reasonable comparison, assume $\lambda$ is direction independent, so that $\lambda(\mathrm{d} u, \mathrm{~d} \theta)=u^{-a-1} \nu(\mathrm{d} \theta)$, where $a \in(0,2)$ is a constant. Then given $\tau$, both approximations use $r_{\tau}=J_{4}(a) \tau^{2 /(2-a)}$ as the cut-off value for jump size and sample $\Delta_{\tau}$, which involves $N_{1} \sim \operatorname{Poisson}\left(a^{-1}\left(r_{\tau}^{-a}-r_{0}^{-a}\right) \nu(S)\right)$ events. However, the PGN approximation also samples $Y_{\tau}$, which involves another $N_{2} \sim \operatorname{Poisson}\left(J(a) r_{\tau}^{-a} \nu(S)\right)$ events. As $\tau \rightarrow 0, N_{2}=O_{p}\left(N_{1}\right)$, and hence the approximations have the same order of complexity. On the other hand, by Theorem 2, the $d_{\mathrm{TV}}$ between $X$ and $\Delta_{\tau}+T_{\tau}$ is $O\left(\tau^{8 a /(2-a)}\right)$, whereas the $d_{\mathrm{TV}}$ between $X$ and its normal approximation is $O\left(\tau^{2 a /(2-a)}\right)$. Therefore, at least asymptotically, the PGN approximation has higher but the same order of computational complexity as the normal approximation, and the extra complexity may lead to significant improvement in error rate when $a$ is not too small, e.g., $a>1 / 8$. 


\section{Proofs of main results}

\subsection{Univariate case}

To prove Theorem 1, we can assume that

$$
\int_{0}^{\infty} t^{2(q+1)} e^{-2 L(t, r)} \mathrm{d} t<\infty
$$

Otherwise, $Q_{q+1}=\infty$ and the result is trivial. We need the following two lemmas.

Lemma 1. 1) Let $\xi$ be i.d. with $\Psi_{\xi}(t)=\int\left(1+\mathrm{i} t u-e^{\mathrm{i} t u}\right) \nu(\mathrm{d} u)$ and $\mathbb{E}|\xi|^{j}<\infty$ for all $j \geq 1$. Given $\epsilon>0$, let $Z \sim N\left(0, \epsilon^{2}\right)$ be independent of $\xi$. Then $\psi_{\xi+Z} \in \mathscr{S}(\mathbb{R})$.

2) Under condition (31), $f_{X_{r}} \in C^{q}(\mathbb{R})$, and for $0 \leq j \leq q, f_{X_{r}}^{(j)}(x) \rightarrow 0$ as $|x| \rightarrow \infty$.

The second lemma is as follows. Note that it does not require matching of cumulants.

Lemma 2. Let $T_{r}$ be defined as in Theorem 1, such that $s(r)<1 /(p+3)$ and $\sigma(r)>0$. Fix $\epsilon>0$. Given $A, B \geq 0$ with $A+B=1$, let $W$ be an i.d. random variable with

$$
\Psi_{W}(t)=A \Psi_{X_{r}}(t)+B \Psi_{T_{r}}(t)+\epsilon^{2} t^{2} / 2 .
$$

Let $\xi=W / \nu$, where $\nu=\sqrt{A \kappa_{2, X_{r}}+B \kappa_{2, T_{r}}}$. Then $f_{\xi} \in \mathscr{S}(\mathbb{R})$ and for $j \geq 1$,

$$
\int\left|f_{\xi}^{(j)}(x)\right| \mathrm{d} x \leq j I_{j-1}(r)+I_{j}(r)+\left(1+\epsilon^{2} / \nu^{2}\right) I_{j+1}(r),
$$

where for $j \geq 0, I_{j}(r) \geq 0$ such that

$$
I_{j}(r)^{2}=\nu^{2 j+1}\left[\frac{\Gamma(j+1 / 2)}{2 D(r)^{2 j+1}}+\int_{1 / r}^{\infty} t^{2 j} e^{-2 H(t, r)} \mathrm{d} t\right],
$$

with

$$
\begin{aligned}
D(r)^{2} & =A C_{1}^{2} \kappa_{2, X_{r}}+B\left(C_{1}^{2} C_{2} \kappa_{2, Y_{r}}+\sigma(r)^{2}\right), \\
H(t, r) & =\frac{A C_{1}^{2} t^{2}}{2} \int_{u<1 /|t|} u^{2} \lambda(\mathrm{d} u)+\frac{B \sigma(r)^{2} t^{2}}{2} .
\end{aligned}
$$

To prove Theorem 1 , by $d_{\mathrm{TV}}\left(X, \Delta_{r}+T_{r}\right)=d_{\mathrm{TV}}\left(\Delta_{r}+X_{r}, \Delta_{r}+T_{r}\right) \leq d_{\mathrm{TV}}\left(X_{r}, T_{r}\right)$, it suffices to show (13) for $d_{\mathrm{TV}}\left(X_{r}, T_{r}\right)$. Let $Z$ and $Z^{\prime}$ be i.i.d. $N(0,1)$ random variables independent of $X_{r}$ and $T_{r}$. Fix $\epsilon>0$. Letting $h$ be a measurable function with $\|h\|_{\infty} \leq 1$, our first goal is to bound

$$
\Delta_{\epsilon}=\mathbb{E}\left[h\left(X_{r}+\epsilon Z\right)-h\left(T_{r}+\epsilon Z^{\prime}\right)\right] .
$$

For $n \geq 2$, we have representations

$$
X_{r}+\epsilon Z=U_{2}+\cdots+U_{n+1}, \quad T_{r}+\epsilon Z^{\prime}=V_{1}+\cdots+V_{n},
$$

(note the index of $U$ starts at 2), where $U_{i}$ and $V_{j}, i, j=1, \ldots, n+1$, are independent i.d. random variables with

$$
\Psi_{U_{i}}(t)=n^{-1} \Psi_{X_{r}+\epsilon Z}(t), \quad \Psi_{V_{i}}(t)=n^{-1} \Psi_{T_{r}+\epsilon Z^{\prime}}(t)
$$


For $k=1, \ldots, n+1$, let

$$
W_{k}=\sum_{1 \leq j<k} V_{j}+\sum_{k<j \leq n+1} U_{j}
$$

and $g_{k}(x)=\mathbb{E} h\left(W_{k}+x\right)$. By $X_{r}+\epsilon Z=W_{1}$ and $T_{r}+\epsilon Z^{\prime}=W_{n+1}$, it is clear that

$$
\begin{aligned}
\left|\Delta_{\epsilon}\right| & =\left|g_{1}(0)-g_{n+1}(0)\right| \\
& \leq\left|\mathbb{E}\left[g_{1}\left(U_{1}\right)-g_{n+1}\left(V_{n+1}\right)\right]\right|+\left|\mathbb{E}\left[g_{1}\left(U_{1}\right)-g_{1}(0)\right]\right|+\left|\mathbb{E}\left[g_{n+1}\left(V_{n+1}\right)-g_{n+1}(0)\right]\right| .
\end{aligned}
$$

We bound the expectations on the last line separately. By $W_{k}+V_{k}=W_{k+1}+U_{k+1}$,

$$
h\left(W_{1}+U_{1}\right)-h\left(W_{n+1}+V_{n+1}\right)=\sum_{k=1}^{n+1}\left[h\left(W_{k}+U_{k}\right)-h\left(W_{k}+V_{k}\right)\right] .
$$

By independence, $\mathbb{E} h\left(W_{k}+U_{k}\right)=\mathbb{E} g_{k}\left(U_{k}\right)$ and $\mathbb{E} h\left(W_{k}+V_{k}\right)=\mathbb{E} g_{k}\left(V_{k}\right)$. Therefore, taking expectation on both sides of the displayed identity yields

$$
\mathbb{E}\left[g_{1}\left(U_{1}\right)-g_{n+1}\left(V_{n+1}\right)\right]=\sum_{k=1}^{n+1} \mathbb{E}\left[g_{k}\left(U_{k}\right)-g_{k}\left(V_{k}\right)\right]
$$

Denote $\nu=\kappa_{2, X_{r}}^{1 / 2}$. Let $\xi_{k}=W_{k} / \nu$. By Lemma $1, f_{\xi_{k}} \in \mathscr{S}(\mathbb{R})$. As a result,

$$
g_{k}(x)=\int h(\nu u+x) f_{\xi_{k}}(u) \mathrm{d} u=\int h(\nu u) f_{\xi_{k}}(u-x / \nu) \mathrm{d} u
$$

is smooth. By Taylor expansion around 0,

$$
g_{k}\left(U_{k}\right)-g_{k}\left(V_{k}\right)=\sum_{j=1}^{q-1} \frac{g_{k}^{(j)}(0)}{j !}\left(U_{k}^{j}-V_{k}^{j}\right)+\frac{1}{q !}\left[g_{k}^{(q)}\left(\theta\left(U_{k}\right) U_{k}\right) U_{k}^{q}-g_{k}^{(q)}\left(\theta\left(V_{k}\right) V_{k}\right) V_{k}^{q}\right],
$$

where $\theta(x) \in[0,1]$. By assumption, $\kappa_{j, X_{r}}=\kappa_{j, T_{r}}$ for $1 \leq j<q$. Since $\kappa_{j, U_{k}}=n^{-1} \kappa_{j, X_{r}+\epsilon Z}=$ $n^{-1}\left(\kappa_{j, X_{r}}+\epsilon^{2} \mathbf{1}\{j=2\}\right)$, and likewise $\kappa_{j, V_{k}}=n^{-1}\left(\kappa_{j, T_{r}}+\epsilon^{2} \mathbf{1}\{j=2\}\right)$, then $\kappa_{j, U_{k}}=\kappa_{j, V_{k}}$ for $1 \leq j<q$. As a result, $\mathbb{E} U_{k}^{j}=\mathbb{E} V_{k}^{j}$ for $1 \leq j<q$ and hence

$$
\mathbb{E}\left[g_{k}\left(U_{k}\right)-g_{k}\left(V_{k}\right)\right]=\frac{1}{q !} \mathbb{E}\left[g_{k}^{(q)}\left(\theta\left(U_{k}\right) V_{k}\right) U_{k}^{q}-g_{k}^{(q)}\left(\theta\left(V_{k}\right) V_{k}\right) V_{k}^{q}\right]
$$

giving

$$
\left|\mathbb{E}\left[g_{k}\left(U_{k}\right)-g_{k}\left(V_{k}\right)\right]\right| \leq \frac{\left\|g_{k}^{(q)}\right\|_{\infty}}{q !}\left[\mathbb{E}\left|U_{k}\right|^{q}+\mathbb{E}\left|V_{k}\right|^{q}\right] .
$$

Since $g_{k}^{(q)}(x)=(-\nu)^{-q} \int h(\nu u) f_{\xi_{k}}^{(q)}(u-x / \nu) \mathrm{d} u$, then

$$
\left\|g_{k}^{(q)}\right\|_{\infty} \leq \nu^{-q} \int\left|f_{\xi_{k}}^{(q)}(u)\right| \mathrm{d} u .
$$

Because

$$
\begin{aligned}
\Psi_{W_{k}}(t) & =(k-1) \Psi_{V_{1}}(t)+(n+1-k) \Psi_{U_{1}}(t) \\
& =\frac{n+1-k}{n} \Psi_{X_{r}}(t)+\frac{k-1}{n} \Psi_{T_{r}}(t)+\frac{\epsilon^{2} t^{2}}{2},
\end{aligned}
$$


we can apply Lemma 2 with $\nu^{2}=\kappa_{2, X_{r}}=\kappa_{2, T_{r}}, A=(n+1-k) / n$ and $B=(k-1) / n$ therein. By definition of $D(r)$ and $H(t, r)$ in Lemma 2,

$$
D(r)^{2}=A C_{1}^{2} \nu^{2}+B\left(C_{1}^{2} C_{2} \kappa_{2, Y_{r}}+\sigma(r)^{2}\right) \geq A C_{1}^{2} \nu^{2}+B C_{1}^{2} C_{2}\left(\kappa_{2, Y_{r}}+\sigma(r)^{2}\right) \geq C_{1}^{2} C_{2} \nu^{2}
$$

and

$$
\begin{aligned}
H(t, r) & =\frac{A C_{1}^{2} t^{2}}{2} \int_{u<1 /|t|} u^{2} \lambda(\mathrm{d} u)+\frac{B \sigma(r)^{2} t^{2}}{2} \\
& \geq \frac{(A+B) t^{2}}{2} \min \left\{C_{1}^{2} \int_{u<1 /|t|} u^{2} \lambda(\mathrm{d} u), \sigma(r)^{2}\right\}=L(t, r) .
\end{aligned}
$$

By definition of $Q_{j}(r)$ in Theorem 1 and definition of $I_{j}(r)$ in Lemma $2, I_{j}(r)^{2} \leq Q_{j}(r)^{2}$. By condition (31), $Q_{j}(r)^{2}<\infty$ for $0 \leq j \leq q+1$. Thus (35) and Lemma 2 give

$$
\left\|g_{k}^{(q)}\right\|_{\infty} \leq \nu^{-q}\left[q Q_{q-1}(r)+Q_{q}(r)+\left(1+\epsilon^{2} / \nu^{2}\right) Q_{q+1}(r)\right]:=M_{\epsilon}<\infty .
$$

Since $M_{\epsilon}$ is independent of $k$, by (33) and (34),

$$
\left|\mathbb{E} g_{1}\left(U_{1}\right)-\mathbb{E} g_{n+1}\left(V_{n+1}\right)\right| \leq \sum_{k=1}^{n+1}\left|\mathbb{E}\left[g_{k}\left(U_{k}\right)-g_{k}\left(V_{k}\right)\right]\right| \leq \frac{M_{\epsilon}}{q !} \sum_{k=1}^{n+1}\left(\mathbb{E}\left|U_{k}\right|^{q}+\mathbb{E}\left|V_{k}\right|^{q}\right) .
$$

Since the Lévy measure of $X_{r}$ has bounded support, $\mathbb{E}\left|X_{r}+\epsilon Z\right|^{q}<\infty$. Meanwhile, from (5), $\mathbb{E}\left|Y_{r}+\epsilon Z\right|^{q}<\infty$. Then by Lemma 3.1 in [1],

$$
\sum_{k=1}^{n+1} \mathbb{E}\left|U_{k}\right|^{q} \rightarrow|\kappa|_{q, X_{r}+\epsilon Z}=|\kappa|_{q, X_{r}}, \quad \sum_{k=1}^{n+1} \mathbb{E}\left|V_{k}\right|^{q} \rightarrow|\kappa|_{q, T_{r}+\epsilon Z^{\prime}}=|\kappa|_{q, Y_{r}} .
$$

As a result,

$$
\limsup _{n \rightarrow \infty}\left|\mathbb{E} g_{1}\left(U_{1}\right)-\mathbb{E} g_{n+1}\left(V_{n+1}\right)\right| \leq \frac{M_{\epsilon}}{q !}\left(|\kappa|_{q, X_{r}}+|\kappa|_{q, Y_{r}}\right) .
$$

On the other hand, $\left|\mathbb{E}\left[g_{1}\left(U_{1}\right)-g_{1}(0)\right]\right| \leq\left\|g_{1}^{\prime}\right\|_{\infty} \mathbb{E}\left|U_{1}\right|$. Since $\mathbb{E} U_{1}^{2}=\mathbb{V}\left(U_{1}\right)=\mathbb{V}\left(X_{r}+\epsilon Z\right) / n$, by Cauchy-Schwartz inequality, as $n \rightarrow \infty, \mathbb{E}\left|U_{1}\right| \rightarrow 0$. As in (35), $\left\|g_{1}^{\prime}\right\|_{\infty} \leq \nu^{-1} \int\left|f_{\xi_{1}}^{\prime}(u)\right| \mathrm{d} u$. By $f_{\xi_{1}} \in \mathscr{S}(\mathbb{R}),\left\|g_{1}^{\prime}\right\|_{\infty}<\infty$. Since $g_{1}(x)=\mathbb{E} h\left(X_{r}+\epsilon Z+x\right)$ is a function independent of $n$, it follows that $\mathbb{E}\left[g_{1}\left(U_{1}\right)-g_{1}(0)\right] \rightarrow 0$ as $n \rightarrow \infty$. Likewise, $\mathbb{E}\left[g_{n+1}\left(V_{n+1}\right)-g_{n+1}(0)\right] \rightarrow 0$. Together with (32) and (36), this implies

$$
\left|\mathbb{E} h\left(X_{r}+\epsilon Z\right)-\mathbb{E} h\left(T_{r}+\epsilon Z^{\prime}\right)\right| \leq \frac{M_{\epsilon}}{q !}\left(|\kappa|_{q, X_{r}}+|\kappa|_{q, Y_{r}}\right)
$$

Let $G \subset \mathbb{R}$ be the union of a finite number of $\left(a_{i}, b_{i}\right)$ and $h(x)=\mathbf{1}\{x \in G\}$. By 2) of Lemma $1, \mathbb{P}\left\{X_{r}=a_{i}\right.$ or $b_{i}$, some $\left.i\right\}=0$. Then $h\left(X_{r}+\epsilon Z\right)-h\left(X_{r}\right) \rightarrow 0$ a.s. as $\epsilon \rightarrow 0+$. On the other hand, since $T_{r}$ is the sum of $Y_{r}$ and an independent nonzero Gaussian random variable, by 1$)$ of Lemma $1, f_{T_{r}} \in \mathscr{S}(\mathbb{R})$. As a result, $h\left(T_{r}+\epsilon Z^{\prime}\right)-h\left(T_{r}\right) \rightarrow 0$ a.s. as $\epsilon \rightarrow 0+$. Also, $M_{\epsilon} \rightarrow M:=\nu^{-q}\left[q Q_{q-1}(r)+Q_{q}(r)+Q_{q+1}(r)\right]$. Thus, by dominated convergence,

$$
\left|\mathbb{P}\left\{X_{r} \in G\right\}-\mathbb{P}\left\{T_{r} \in G\right\}\right| \leq \frac{M}{q !}\left(|\kappa|_{q, X_{r}}+|\kappa|_{q, Y_{r}}\right) .
$$


Let $A \subset \mathbb{R}$ be measurable. Given $\delta>0$, there is $R>0$, such that, letting $B=A \cap(-R, R)$, $\mathbb{P}\left\{X_{r} \in A \backslash B\right\}+\mathbb{P}\left\{T_{r} \in A \backslash B\right\}<\delta$. Then there is an open $G \supset B$ with $\ell(G \backslash B)<\delta$, where $\ell$ is the Lebesgue measure. $G$ is the union of at most countably many disjoint open intervals $\left(a_{i}, b_{i}\right)$. Let $G_{k}=\cup_{i=1}^{k}\left(a_{i}, b_{i}\right)$. Then

$$
\begin{aligned}
&\left|\mathbb{P}\left\{X_{r} \in A\right\}-\mathbb{P}\left\{T_{r} \in A\right\}\right| \\
& \leq\left|\mathbb{P}\left\{X_{r} \in G_{k}\right\}-\mathbb{P}\left\{T_{r} \in G_{k}\right\}\right|+\mathbb{P}\left\{X_{r} \in G \backslash G_{k}\right\}+\mathbb{P}\left\{T_{r} \in G \backslash G_{k}\right\} \\
&+\mathbb{P}\left\{X_{r} \in A \backslash G\right\}+\mathbb{P}\left\{T_{r} \in A \backslash G\right\}+\mathbb{P}\left\{X_{r} \in G \backslash A\right\}+\mathbb{P}\left\{T_{r} \in G \backslash A\right\} \\
& \leq \frac{M}{q !}\left(|\kappa|_{q, X_{r}}+|\kappa|_{q, Y_{r}}\right)+\mathbb{P}\left\{X_{r} \in G \backslash G_{k}\right\}+\mathbb{P}\left\{T_{r} \in G \backslash G_{k}\right\} \\
&+\mathbb{P}\left\{X_{r} \in A \backslash B\right\}+\mathbb{P}\left\{T_{r} \in A \backslash B\right\}+\left(\left\|f_{X_{r}}\right\|_{\infty}+\left\|f_{T_{r}}\right\|_{\infty}\right) \ell(G \backslash B) \\
& \leq \frac{M}{q !}\left(|\kappa|_{q, X_{r}}+|\kappa|_{q, Y_{r}}\right)+\mathbb{P}\left\{X_{r} \in G \backslash G_{k}\right\}+\mathbb{P}\left\{T_{r} \in G \backslash G_{k}\right\} \\
&+\left(1+\left\|f_{X_{r}}\right\|_{\infty}+\left\|f_{T_{r}}\right\|_{\infty}\right) \delta .
\end{aligned}
$$

By Lemma 1, $\left\|f_{X_{r}}\right\|_{\infty}+\left\|f_{T_{r}}\right\|_{\infty}<\infty$. Then, letting $k \rightarrow \infty$ followed by $\delta \rightarrow 0$ yields

$$
\left.\mid \mathbb{P}\left\{X_{r} \in A\right\}-\mathbb{P}\left\{T_{r} \in A\right\}\right] \mid \leq \frac{M}{q !}\left(|\kappa|_{q, X_{r}}+|\kappa|_{q, Y_{r}}\right) .
$$

This completes the proof of Theorem 1 .

\subsection{Multivariate case}

We shall prove Theorem 2 by standardizing the random variables involved. First, we have the following simple result.

Lemma 3. Let $X$ be i.d. with Lévy measure $\lambda(\mathrm{d} u, \mathrm{~d} \theta)$ in polar coordinates and $A$ be a nonsingular matrix. Then $A^{-1} X$ has Lévy measure $\lambda\left(J_{A}^{-1}(\cdot)\right)$ and variance $A^{-1} \mathbb{V}(X) A^{-1}$, where

$$
J_{A}(u, \theta)=\left(\left\|A^{-1} \theta\right\| u, \frac{A^{-1} \theta}{\left\|A^{-1} \theta\right\|}\right), \quad(u, \theta) \in(0, \infty) \times S .
$$

Furthermore, $J_{A}^{-1}(u, \theta)=J_{A^{-1}}(u, \theta)=(\|A \theta\| u, A \theta /\|A \theta\|)$.

Proof. Denote $h(z)=1+z-e^{z}$. For any $t \in \mathbb{R}^{d}$,

$$
\begin{aligned}
\Psi_{A^{-1} X}(t)=\Psi_{X}\left(A^{-1} t\right) & =\int h\left(\mathrm{i}\left\langle A^{-1} t, \theta\right\rangle u\right) \lambda(\mathrm{d} u, \mathrm{~d} \theta) \\
& =\int h\left(\mathrm{i}\left\langle t, A^{-1} \theta\right\rangle u\right) \lambda(\mathrm{d} u, \mathrm{~d} \theta)=\int h(\mathrm{i}\langle t, \omega\rangle v) \lambda(\mathrm{d} u, \mathrm{~d} \theta),
\end{aligned}
$$

where $(v, \omega)=J_{A}(u, \theta)$. Then the lemma easily follows.

Recall $A_{\tau}=\Sigma_{\tau}^{1 / 2}$ and $K^{*}=\tau^{2} A_{\tau}^{-1} K_{\nu} A_{\tau}^{-1}$, where $\Sigma_{\tau}=\mathbb{V}\left(X_{\tau}\right)=\mathbb{V}\left(T_{\tau}\right)$. Let

$$
X^{*}=A_{\tau}^{-1} X_{\tau}, \quad T^{*}=A_{\tau}^{-1} T_{\tau}, \quad Y^{*}=A_{\tau}^{-1} Y_{\tau} .
$$

Then $X^{*}$ and $T^{*}$ are standardized, i.e., $\mathbb{E} X^{*}=\mathbb{E} T^{*}=0$ and $\mathbb{V}\left(X^{*}\right)=\mathbb{V}\left(T^{*}\right)=I$. It is easy to check that if $\kappa_{\alpha, X_{\tau}}=\kappa_{\alpha, T_{\tau}}$ for $2 \leq|\alpha|<q$, then $\kappa_{\alpha, X^{*}}=\kappa_{\alpha, T^{*}}$ for $2 \leq|\alpha|<q$. By Lemma 3, $X^{*}$ and $Y^{*}$ have Lévy measures $\lambda^{*}(\mathrm{~d} u, \mathrm{~d} \theta)=\lambda_{\tau}(\mathrm{d} v, \mathrm{~d} \omega)$ and $\gamma^{*}(\mathrm{~d} u, \mathrm{~d} \theta)=\gamma_{\tau}(\mathrm{d} v, \mathrm{~d} \omega)$, respectively, where $(v, \omega)=J_{A_{\tau}}^{-1}(u, \theta)$, and $T^{*}=Y^{*}+Z^{*}$, where $Z^{*} \sim N\left(0, K^{*}\right)$ is independent of $Y^{*}$. 
Lemma 4. Under conditions (24) and (25), $\mathbb{E}\left\|X^{*}\right\|^{a}<\infty$ and $\mathbb{E}\left\|Y^{*}\right\|^{a}<\infty$ for any $a>0$.

The next lemma is analogous to Lemma 1.

Lemma 5. Given $\tau>0$, the following statements are true.

1) Under condition (24) and (25), for any $a \geq 0$ and $b \geq 0$ with $a+b>0$ and $\epsilon>0$, if $\xi$ is i.d. with $\Psi_{\xi}=a \Psi_{X^{*}}+b \Psi_{Y^{*}}$ and $Z \sim N\left(0, \epsilon^{2} I\right)$ is independent of $\xi$, then $\psi_{\xi+Z} \in \mathscr{S}\left(\mathbb{R}^{d}\right)$.

2) Under condition (26), $f_{X^{*}} \in C^{q}\left(\mathbb{R}^{d}\right)$ and for each $|\alpha| \leq q, f_{X^{*}}^{(\alpha)}(x) \rightarrow 0$ as $|x| \rightarrow \infty$.

The next lemma is analogous to Lemma 2.

Lemma 6. Given $\epsilon>0$ and $A, B \geq 0$ with $A+B=1$, let $\xi$ be an i.d. random variable with

$$
\Psi_{\xi}(t)=A \Psi_{X^{*}}(t)+B \Psi_{T^{*}}(t)+\epsilon^{2}\|t\|^{2} / 2 .
$$

Then, under condition (25), for $m \geq 3$,

$$
\max _{|\alpha|=m} \int\left|f_{\xi}^{(\alpha)}(x)\right| \mathrm{d} x \leq\left(1+\epsilon^{2} / d\right)^{h(d)} c(d, m) \sqrt{1+\int_{1 / R^{*}}^{\infty} L_{d, m}(s) e^{-\varrho_{\tau}(1 / s) s^{2}} \mathrm{~d} s,}
$$

where $c(d, m)$ is a constant only depending on $(d, m)$ and $L_{d, m}(s)$ a polynomial of order no greater than $2 m+2 h(d)+d-1$ whose coefficients are constants only depending on $(d, m)$.

The proof of Theorem 2 is similar to the one for Theorem 1, so we will only give its sketch. By $d_{\mathrm{TV}}\left(X, \Delta_{\tau}+T_{\tau}\right) \leq d_{\mathrm{TV}}\left(X_{\tau}, T_{\tau}\right)=d_{\mathrm{TV}}\left(X^{*}, T^{*}\right)$, it suffices to show (27) for $d_{\mathrm{TV}}\left(X^{*}, T^{*}\right)$. Let $Z$ and $Z^{\prime} \in \mathbb{R}^{d}$ be i.i.d. $N(0, I)$ random variables independent of $X^{*}$ and $T^{*}$. Given $\epsilon>0$, for $n \geq 2$,

$$
X^{*}+\epsilon Z=U_{2}+\cdots+U_{n+1}, \quad T^{*}+\epsilon Z^{\prime}=V_{1}+\cdots+V_{n},
$$

where $U_{i}$ and $V_{j}$ are independent and i.d. with $\Psi_{U_{i}}=n^{-1} \Psi_{X_{r}^{*}+\epsilon Z}$ and $\Psi_{V_{j}}=n^{-1} \Psi_{T_{r}^{*}+\epsilon Z^{\prime}}$. Let $W_{k}=V_{1}+\cdots+V_{k-1}+U_{k+1}+\cdots+U_{n+1}$. Given a measurable function $h$ with $\|h\|_{\infty} \leq 1$, let $g_{k}(x)=\mathbb{E} h\left(W_{k}+x\right)$. Then

$$
\begin{aligned}
& \left|\mathbb{E}\left[h\left(X^{*}+\epsilon Z\right)-h\left(Y^{*}+\epsilon Z^{\prime}\right)\right]\right| \\
& \leq \sum_{k=1}^{n+1}\left|\mathbb{E}\left[g_{k}\left(U_{k}\right)-g_{k}\left(V_{k}\right)\right]\right|+\left|\mathbb{E}\left[g_{1}\left(U_{1}\right)-g_{1}(0)\right]\right|+\left|\mathbb{E}\left[g_{n+1}\left(V_{n+1}\right)-g_{n+1}(0)\right]\right|
\end{aligned}
$$

Since $g_{k}(x)=\int h(u) f_{W_{k}}(u-x) \mathrm{d} u$ and $\|h\|_{\infty} \leq 1$, by Lemma $5, g_{k} \in C^{\infty}$. Then

$$
g_{k}\left(U_{k}\right)-g_{k}\left(V_{k}\right)=\sum_{|\alpha|<q} \frac{g_{k}^{(\alpha)}(0)}{\alpha !}\left(U_{k}^{\alpha}-V_{k}^{\alpha}\right)+\sum_{|\alpha|=q} \frac{1}{\alpha !}\left[g_{k}^{(\alpha)}\left(\theta\left(U_{k}\right) U_{k}\right) U_{k}^{\alpha}-g_{k}^{(\alpha)}\left(\theta\left(V_{k}\right) V_{k}\right) V_{k}^{\alpha}\right],
$$

where $\theta(x) \in[0,1]$. Since $\mathbb{E} U_{k}^{\alpha}=\mathbb{E} V_{k}^{\alpha}$ for $2 \leq|\alpha|<q$, then

$$
\left|\mathbb{E}\left[g_{k}\left(U_{k}\right)-g_{k}\left(V_{k}\right)\right]\right| \leq \sum_{|\alpha|=q} \frac{\left\|g_{k}^{(\alpha)}\right\|_{\infty}}{\alpha !}\left[\mathbb{E}\left|U_{k}^{\alpha}\right|+\mathbb{E}\left|V_{k}^{\alpha}\right|\right] .
$$

By Lemma 6, for $|\alpha|=q$,

$$
\left\|g_{k}^{(\alpha)}\right\|_{\infty} \leq \int\left|f_{W_{k}}^{(\alpha)}(u)\right| \mathrm{d} u \leq\left(1+\epsilon^{2} / d\right)^{h(d)} G(d, q, \tau),
$$


which is finite by (26). Note $\left|u^{\alpha}\right| \leq\|u\|^{q}$. Then by Lemma 4 and Proposition 1 , as $n \rightarrow \infty$,

$$
\sum_{k=1}^{n+1} \mathbb{E}\left|U_{k}^{(\alpha)}\right| \rightarrow|\kappa|_{\alpha, X^{*}}, \quad \sum_{k=1}^{n+1} \mathbb{E}\left|V_{k}^{(\alpha)}\right| \rightarrow|\kappa|_{\alpha, Y^{*}} .
$$

From here, an argument similar to that for Theorem 1 leads to

$$
d_{\mathrm{TV}}\left(X^{*}, T^{*}\right) \leq G(d, q, \tau) \sum_{|\alpha|=q} \frac{|\kappa|_{\alpha, X^{*}}+|\kappa|_{\alpha, Y^{*}}}{\alpha !} .
$$

Since

$$
\begin{aligned}
\sum_{|\alpha|=q} \frac{|\kappa|_{\alpha, X^{*}}}{\alpha !} & =\sum_{|\alpha|=q} \frac{1}{\alpha !} \int\left|(u \theta)^{\alpha}\right| \lambda^{*}(\mathrm{~d} u, \mathrm{~d} \theta) \\
& =\sum_{|\alpha|=q} \frac{1}{\alpha !} \int\left|\left(u A_{\tau}^{-1} \theta\right)^{\alpha}\right| \lambda_{\tau}(\mathrm{d} u, \mathrm{~d} \theta)=\frac{1}{q !} \int\left\|\left(u A_{\tau}^{-1} \theta\right)\right\|_{1}^{q} \lambda_{\tau}(\mathrm{d} u, \mathrm{~d} \theta)
\end{aligned}
$$

and a similar expression holds for $\sum_{|\alpha|=q}|\kappa|_{\alpha, T^{*}} / \alpha$ !, the proof of Theorem 2 is then complete.

Proof of Proposition \%. 1) By $\tau^{2} K_{\nu} \leq \Sigma_{\tau}$, the smallest eigenvalue of $\Sigma_{\tau}$ is at least $\tau^{2} c\left(K_{\nu}\right)^{2}$, yielding the first assertion and $R^{*}=O\left(\operatorname{ess} \sup r_{\tau}(\theta) / \tau\right)$. Then by $(28), R^{*} \rightarrow 0$ as $\tau \rightarrow 0+$.

2) By condition (29), for small $\tau>0, \Sigma_{\tau}=\int \theta \theta^{\prime} \nu(\mathrm{d} \theta) \int u^{2} \lambda_{\tau}(\mathrm{d} u \mid \theta) \leq b \tau^{2} K_{\nu}$ and hence

$$
\tau^{2} K_{\nu} \geq c_{1} \Sigma_{\tau}
$$

where $c_{1}=1 / b>0$. Denote

$$
g_{\tau}(s, \theta)=\int u^{2} \mathbf{1}\left\{u<r_{\tau}(\theta) \wedge \frac{1}{\left\|A_{\tau}^{-1} \theta\right\| s}\right\} \lambda(\mathrm{d} u \mid \theta) .
$$

Then $M_{\tau}(1 / s)=\int \theta \theta^{\prime} g_{\tau}(s, \theta) \nu(\mathrm{d} \theta)$. If $1 / R^{*}<s<\frac{1}{\left\|A_{\tau}^{-1} \theta\right\| r_{\tau}(\theta)}$, then by definition of $r_{\tau}(\theta)$,

$$
g_{\tau}(s, \theta)=\int u^{2} \mathbf{1}\left\{u<r_{\tau}(\theta)\right\} \lambda(\mathrm{d} u \mid \theta)=\tau^{2} .
$$

If $s \geq \frac{1}{\left\|A_{\tau}^{-1} \theta\right\| r_{\tau}(\theta)}$, then by $\left\|A_{\tau}^{-1} \theta\right\| \leq 1 /(c \tau)$ and condition (30), with $M$ to be determined,

$$
g_{\tau}(s, \theta)=\int u^{2} \mathbf{1}\left\{u<\frac{1}{\left\|A_{\tau}^{-1} \theta\right\| s}\right\} \lambda(\mathrm{d} u \mid \theta) \geq \int u^{2} \mathbf{1}\left\{u<\frac{c \tau}{s}\right\} \lambda(\mathrm{d} u \mid \theta) \geq \frac{c^{2} \tau^{2} M}{s^{2}} \ln \frac{s}{c \tau},
$$

Thus, for $s>1 / R^{*}, g_{\tau}(s, \theta) \geq \tau^{2} F_{\tau}(s)$, where

$$
F_{\tau}(s)=\min \left\{1, \frac{c^{2} M}{s^{2}} \ln \frac{s}{c \tau}\right\},
$$

and as a result, by (37), $M_{\tau}(1 / s) \geq \tau^{2} F_{\tau}(s) K_{\nu} \geq c_{1} F_{\tau}(s) \Sigma_{\tau}$, giving $A_{\tau}^{-1} M_{\tau}(1 / s) A_{\tau}^{-1} \geq c_{1} F_{\tau}(s)$. Also, by (37), $K^{*}=\tau^{2} A_{\tau}^{-1} K_{\nu} A_{\tau}^{-1} \geq c_{1} I$. Thus, there is $c_{2}=c_{2}\left(b, K_{\nu}\right)>0$, such that for $\tau>0$ small enough and $s>1 / R^{*}$,

$$
\varrho_{\tau}(1 / s) s^{2} \geq c_{2} F_{\tau}(s) s^{2}=c_{2} \min \left\{s^{2}, c^{2} M \ln \frac{s}{c \tau}\right\} .
$$

If $M>0$ is large enough so that $c_{2} c^{2} M>2 q+2 h(d)+d$, then

$$
\int_{1 / R^{*}}^{\infty} s^{2 q+2 h(d)+d-1} e^{-\varrho_{\tau}(1 / s) s^{2}} \mathrm{~d} s \leq \int_{1 / R^{*}}^{\infty} s^{2 q+2 h(d)+d-1}\left\{e^{-c_{2} s^{2}}+\left(\frac{s}{c \tau}\right)^{-c_{2} c^{2} M}\right\} \mathrm{d} s
$$

is $o(1)$ as $\tau \rightarrow 0$. It then easily follows that $G(d, q, \tau)=c(d, q)+o(1)$. 


\section{Proofs of auxiliary results}

\subsection{Proposition 1}

Given $0<a<b<\infty$, let $0 \leq h(u) \leq 1$ be a continuous function with compact support in $\mathbb{R}^{d} \backslash\{0\}$, such that $h(u)=1$ if $\|u\| \in[a, b]$. As pointed out in Section 2.2,

$$
\begin{aligned}
& \lim _{n \rightarrow \infty} n \mathbb{E}\left[h\left(U_{n}\right) f\left(U_{n}\right)\right]=\int h(u) f(u) \lambda(\mathrm{d} u)<\infty, \\
& \lim _{n \rightarrow \infty} n \mathbb{E}\left[h\left(U_{n}\right) g\left(\left\|U_{n}\right\|\right)\right]=\int h(u) g(\|u\|) \lambda(\mathrm{d} u)<\infty .
\end{aligned}
$$

From the second line and monotone convergence it follows that

$$
\liminf _{n \rightarrow \infty} n \mathbb{E}\left[g\left(\left\|U_{n}\right\|\right)\right] \geq \int g(\|u\|) \lambda(\mathrm{d} u) .
$$

Clearly, $f \in L^{1}(\lambda)$ if $g(\|u\|) \in L^{1}(\lambda)$. Furthermore,

$$
\begin{aligned}
\left|n \mathbb{E}\left[f\left(U_{n}\right)\right]-n \mathbb{E}\left[h\left(U_{n}\right) f\left(U_{n}\right)\right]\right| & \leq n \mathbb{E}\left[\left(1-h\left(U_{n}\right)\right) g\left(\left\|U_{n}\right\|\right)\right] \\
& \leq n \mathbb{E}\left[g\left(\left\|U_{n}\right\|\right) \mathbf{1}\left\{\left\|U_{n}\right\| \leq a \text { or }\left\|U_{n}\right\| \geq b\right\}\right] .
\end{aligned}
$$

Therefore, to prove the lemma, it suffices to show

$$
\begin{aligned}
& \limsup _{n \rightarrow \infty} n \mathbb{E}\left[g\left(\left\|U_{n}\right\|\right) \mathbf{1}\left\{\left\|U_{n}\right\| \leq a\right\}\right] \rightarrow 0, \quad \text { as } a \rightarrow 0+, \\
& \limsup _{n \rightarrow \infty} n \mathbb{E}\left[g\left(\left\|U_{n}\right\|\right) \mathbf{1}\left\{\left\|U_{n}\right\| \geq b\right\}\right] \rightarrow 0, \quad \text { as } b \rightarrow \infty .
\end{aligned}
$$

Let $m(a)=\sup _{0<\|t\| \leq a} g(t) / t^{2}$. For $n \geq 1$, since $\mathbb{V}\left(U_{n}\right)=\mathbb{V}(X) / n$ and $\mathbb{E} U_{n}=\mathbb{E} X / n$,

$$
\begin{aligned}
n \mathbb{E}\left[\mid g\left(\left\|U_{n}\right\|\right) \mathbf{1}\left\{\left\|U_{n}\right\| \leq a\right\}\right] & \leq m(a) n \mathbb{E}\left[\left\|U_{n}\right\|^{2}\right] \\
& =m(a) n\left[\operatorname{tr}\left(\mathbb{V}\left(U_{n}\right)\right)+\left\|\mathbb{E} U_{n}\right\|^{2}\right] \\
& =m(a)\left[\operatorname{tr}(\mathbb{V}(X))+\|\mathbb{E} X\|^{2} / n\right] .
\end{aligned}
$$

Since $m(a) \rightarrow 0$, then (38) follows. Next, since $g$ is continuous and non-decreasing,

$$
\begin{aligned}
n \mathbb{E}\left[g\left(\left\|U_{n}\right\|\right) \mathbf{1}\left\{\left\|U_{n}\right\| \geq b\right\}\right] & =\int_{0}^{\infty} n \mathbb{P}\left\{g\left(\left\|U_{n}\right\|\right) \geq s,\left\|U_{n}\right\| \geq b\right\} \mathrm{d} s \\
& =\int_{0}^{\infty} n \mathbb{P}\left\{\left\|U_{n}\right\| \geq g^{*}(s) \vee b\right\} \mathrm{d} s,
\end{aligned}
$$

where $g^{*}(s)=\inf \{x: g(x) \geq s\}$. By Markov inequality,

$$
n \mathbb{P}\left\{\left\|U_{n}\right\| \geq x\right\} \leq \frac{n \mathbb{E}\left\|U_{n}\right\|^{2}}{x^{2}}=\frac{\operatorname{tr}(\mathbb{V}(X))+\|\mathbb{E} X\|^{2} / n}{x^{2}} .
$$

Therefore, for large $x>0, n \mathbb{P}\left\{\left\|U_{n}\right\| \geq x\right\} \leq 1$. Since $c_{0} t \leq 1-(1-t / n)^{n}$ for $t \in[0,1]$, where $c_{0}>0$ is a universal constant, then

$$
c_{0} n \mathbb{P}\left\{\left\|U_{n}\right\| \geq x\right\} \leq 1-\left(1-\mathbb{P}\left\{\left\|U_{n}\right\| \geq x\right\}\right)^{n}=\mathbb{P}\left\{\max _{1 \leq k \leq n}\left\|U_{n, k}\right\| \geq x\right\}
$$


where $U_{n, k}$ are i.i.d. $\sim U_{n}$.

First, suppose $X$ is asymmetric. Let $V_{n 1}, \ldots, V_{n n} \sim U_{n}$ be another set of i.i.d. random variables which are also independent of $U_{n k}$. Fix $\delta \in(0,1)$. By setting $b>0$ even larger, for all $x \geq b$,

$$
\mathbb{P}\left\{\max _{1 \leq k \leq n}\left\|V_{n k}\right\|<\delta x\right\} \geq 1-n \mathbb{P}\left\{\left\|U_{n}\right\| \geq \delta x\right\} \geq 1 / 2 .
$$

As a result,

$$
\begin{aligned}
c_{0} n \mathbb{P}\left\{\left\|U_{n}\right\| \geq x\right\} & \leq 2 \mathbb{P}\left\{\max _{1 \leq k \leq n}\left\|U_{n k}\right\| \geq x\right\} \mathbb{P}\left\{\max _{1 \leq k \leq n}\left\|V_{n k}\right\|<\delta x\right\} \\
& \leq 2 \mathbb{P}\left\{\max _{1 \leq k \leq n}\left\|U_{n k}-V_{n k}\right\| \geq(1-\delta) x\right\} \leq 4 \mathbb{P}\left\{\left\|X^{\prime}\right\| \geq(1-\delta) x\right\}
\end{aligned}
$$

where the last line is due to the symmetry of $U_{n k}-V_{n k}$ and [25], Proposition 2.3. Thus, by (40),

$$
\begin{aligned}
n \mathbb{E}\left[g\left(\left\|U_{n}\right\|\right) \mathbf{1}\left\{\left\|U_{n}\right\| \geq b\right\}\right] & \leq \frac{4}{c_{0}} \int_{0}^{\infty} \mathbb{P}\left\{\frac{\left\|X^{\prime}\right\|}{1-\delta} \geq g^{*}(s) \vee b\right\} \mathrm{d} s \\
& =\frac{4}{c_{0}} \int_{0}^{\infty} \mathbb{P}\left\{g\left(\frac{\left\|X^{\prime}\right\|}{1-\delta}\right) \geq s, \frac{\left\|X^{\prime}\right\|}{1-\delta} \geq b\right\} \mathrm{d} s \\
& =\frac{4}{c_{0}} \mathbb{E}\left[g\left(\frac{\left\|X^{\prime}\right\|}{1-\delta}\right) \mathbf{1}\left\{\left\|X^{\prime}\right\| \geq(1-\delta) b\right\}\right] .
\end{aligned}
$$

If $\mathbb{E}\left[g\left(c\left\|X^{\prime}\right\|\right)\right]<\infty$ for some $c>1$, then by choosing $\delta>0$ with $1 /(1-\delta)<c$, it is seen (39) holds. Moreover, since $g(\|x-y\|) \leq g(2\|x\|)+g(2\|y\|), \mathbb{E}\left[g\left(c\left\|X^{\prime}\right\|\right)\right] \leq 2 \mathbb{E}[g(2 c\|X\|)]$, and hence (39) holds once $\mathbb{E}[g(2 c\|X\|)]<\infty$.

Finally, if $X$ is symmetric, then $U_{n}$ is symmetric and from (41) and [25], Proposition 2.3, $c_{0} n \mathbb{P}\left\{\left\|U_{n}\right\| \geq x\right\} \leq 2 \mathbb{P}\{\|X\| \geq x\}$. Then by similar argument, (39) holds once $\mathbb{E}[g(\|X\|)]<\infty$.

\subsection{Lemmas for univariate case}

Proof of Lemma 1. 1) From the assumption, $\int|u|^{j} \lambda(\mathrm{d} u)<\infty$ for all $j \geq 2$. Then by dominated convergence, $\Psi_{\xi} \in C^{\infty}(\mathbb{R})$ with $\Psi_{\xi}^{(j)}(t)=\int\left(\mathbf{1}\{j=1\}-e^{\mathrm{i} t u}\right)(\mathrm{i} u)^{j} \nu(\mathrm{d} u)$ for $j \geq 1$. By $\left|1-e^{\mathrm{i} x}\right| \leq|x|$ for $x \in \mathbb{R},\left|\Psi_{\xi}^{\prime}(t)\right| \leq \kappa_{2, \xi}|t|$. Clearly, $\left|\Psi_{\xi}^{(j)}(t)\right| \leq|\kappa|_{j, \xi}$ for $j \geq 2$. Since $\psi_{\xi+Z}(t)=\exp \left(-\Psi_{\xi}(t)-\right.$ $\left.\epsilon^{2} t^{2} / 2\right)$, then for $j \geq 0, \psi_{\xi+Z}^{(j)}(t)=P_{j}\left(\Psi_{\xi}^{\prime}(t), \ldots, \Psi_{\xi}^{(j)}(t), t\right) \psi_{\xi}(t) \exp \left(-\epsilon^{2} t^{2} / 2\right)$, where $P_{j}(z)$ is a multivariate polynomial in $z=\left(z_{1}, \ldots, z_{j+1}\right)$ of order $j$. It follows that $\left|\psi_{\xi+Z}^{(j)}(t)\right|=O\left(|t|^{j} e^{-\epsilon^{2} t^{2} / 2}\right)$ and hence for any $p \geq 0,|t|^{p}\left|\psi_{\xi+Z}^{(j)}(t)\right| \rightarrow 0$ as $|t| \rightarrow \infty$, which yields the proof.

2) For $|t| \geq 1 / r$,

$$
\operatorname{Re}\left[\Psi_{X_{r}}(t)\right]=\int_{|u|<r}(1-\cos t u) \lambda(\mathrm{d} u) \geq \int_{|u|<1 /|t|}(1-\cos t u) \lambda(\mathrm{d} u) .
$$

Then by $1-\cos x \geq C_{1} x^{2} / 2$ for $|x| \leq 1$,

$$
\operatorname{Re}\left[\Psi_{X_{r}}(t)\right] \geq \frac{C_{1}^{2} t^{2}}{2} \int_{u<1 /|t|} u^{2} \lambda(\mathrm{d} u) \geq L(t, r) .
$$


On the other hand, by Cauchy-Schwartz inequality,

$$
\begin{aligned}
\int_{|t| \geq 1 / r}|t|^{q}\left|\psi_{X_{r}}(t)\right| \mathrm{d} t & \leq\left(\int \frac{\mathrm{d} t}{1+t^{2}}\right)^{1 / 2}\left(\int_{|t| \geq 1 / r}\left(1+t^{2}\right) t^{2 q}\left|\psi_{X_{r}}(t)\right|^{2} \mathrm{~d} t\right)^{1 / 2} \\
& \leq \sqrt{\pi}\left(\int\left(1+t^{2}\right) t^{2 q} e^{-2 L(t, r)} \mathrm{d} t\right)^{1 / 2}
\end{aligned}
$$

Then by (31), $|t|^{q}\left|\psi_{X_{r}}(t)\right| \in L^{1}(\mathbb{R})$ and the proof follows from Proposition 28.1 of [31].

To prove Lemma 2, we need a type of inequalities that are known (cf. [5], Lemma 11.6). Since the expression of $(\widehat{f})^{(j)}$ becomes complicated rapidly as $j$ increases, the following specific form is used to reduce the maximum order of derivative involved.

Lemma 7. Let $f \in \mathscr{S}(\mathbb{R})$ and $\psi(t)=\widehat{f}$. Then for $j \geq 1$,

$$
\begin{aligned}
& \int\left|f^{(j)}(x)\right| \mathrm{d} x \\
& \leq \frac{1}{\sqrt{2}}\left[\left(\int\left|t^{j} \psi(t)\right|^{2} \mathrm{~d} t\right)^{1 / 2}+j\left(\int\left|t^{j-1} \psi(t)\right|^{2} \mathrm{~d} t\right)^{1 / 2}+\left(\int\left|t^{j} \psi^{\prime}(t)\right|^{2} \mathrm{~d} t\right)^{1 / 2}\right] .
\end{aligned}
$$

Proof. By Cauchy-Schwartz and Minkowski inequalities,

$$
\begin{aligned}
\int\left|f^{(j)}(x)\right| \mathrm{d} x & \leq\left(\int \frac{\mathrm{d} x}{1+x^{2}}\right)^{1 / 2}\left(\int\left|f^{(j)}(x)\right|^{2}\left(1+x^{2}\right) \mathrm{d} x\right)^{1 / 2} \\
& \leq \sqrt{\pi}\left[\left(\int\left|f^{(j)}(x)\right|^{2} \mathrm{~d} x\right)^{1 / 2}+\left(\int\left|x f^{(j)}(x)\right|^{2} \mathrm{~d} x\right)^{1 / 2}\right] \\
& =\frac{1}{\sqrt{2}}\left[\left(\int\left|t^{j} \psi(t)\right|^{2} \mathrm{~d} t\right)^{1 / 2}+\left(\int\left|\left(t^{j} \psi(t)\right)^{\prime}\right|^{2} \mathrm{~d} t\right)^{1 / 2}\right],
\end{aligned}
$$

where the last line follows from Plancherel theorem and the fact that Fourier transforms of $f^{(j)}(x)$ and $x^{j} f(x)$ are $(-i t)^{j} \psi(t)$ and $(-i)^{j} \psi^{(j)}(t)$, respectively ([18], p. 100-102). The proof is complete by applying Minkowski inequality to the last integral.

Proof of Lemma 2. We only consider the case where $\operatorname{sppt}(\lambda) \subset \mathbb{R}_{+}$. The proof for the symmetric case is similar. For brevity, write $f=f_{\xi}, \psi=\psi_{\xi}$, and $\Psi=\Psi_{\xi}$. By Lemma $1, f, \psi \in \mathscr{S}(\mathbb{R})$. Write $M=\epsilon^{2}+B \sigma(r)^{2}$. Then

$$
\operatorname{Re}[\Psi(t)]=\operatorname{Re}\left[\Psi_{W}(t / \nu)\right]=\int(1-\cos t u / \nu)\left[A \lambda_{r}(\mathrm{~d} u)+B \gamma_{r}(\mathrm{~d} u)\right]+\frac{M t^{2}}{2 \nu^{2}} .
$$

If $|t| \leq \nu / r$, then $|t u| / \nu \leq 1$ for $0 \leq u<r$. Since $1-\cos x \geq C_{1}^{2} x^{2} / 2$ for $|x| \leq 1$,

$$
\begin{aligned}
\operatorname{Re}[\Psi(t)] & \geq \frac{C_{1}^{2} t^{2}}{2 \nu^{2}} \int_{0}^{r} u^{2}\left[A \lambda_{r}(\mathrm{~d} u)+B \gamma_{r}(\mathrm{~d} u)\right]+\frac{M t^{2}}{2 \nu^{2}} \\
& =\frac{A C_{1}^{2} \kappa_{2, X_{r}} t^{2}}{2 \nu^{2}}+\frac{B C_{1}^{2} m(r) s(r)^{p+3} t^{2}}{2 \nu^{2}} \int_{0}^{r / s(r)} u^{p+2} e^{-u} \mathrm{~d} u+\frac{M t^{2}}{2 \nu^{2}} .
\end{aligned}
$$


Since $s(r)<r /(p+3)$,

$$
\int_{0}^{r / s(r)} u^{p+2} e^{-u} \mathrm{~d} u \geq \int_{0}^{p+3} u^{p+2} e^{-u} \mathrm{~d} u \geq C_{2} \Gamma(p+3) .
$$

Then by $\Gamma(p+3) m(r) s(r)^{p+3}=\kappa_{2, Y_{r}}$,

$$
\begin{aligned}
\operatorname{Re}[\Psi(t)] & \geq \frac{A C_{1}^{2} \kappa_{2, X_{r}} t^{2}}{2 \nu^{2}}+\frac{B C_{1}^{2} m(r) s(r)^{p+3} C_{2} \Gamma(p+3) t^{2}}{2 \nu^{2}}+\frac{M t^{2}}{2 \nu^{2}} \\
& \geq \frac{A C_{1}^{2} \kappa_{2, X_{r}} t^{2}}{2 \nu^{2}}+\frac{B C_{1}^{2} C_{2} \kappa_{2, Y_{r}} t^{2}}{2 \nu^{2}}+\frac{B \sigma(r)^{2} t^{2}}{2 \nu^{2}}=\frac{D(r)^{2} t^{2}}{2 \nu^{2}} .
\end{aligned}
$$

If $|t|>\nu / r$, then $r>\nu /|t|$ and

$$
\operatorname{Re}[\Psi(t)] \geq \frac{A C_{1}^{2} t^{2}}{2 \nu^{2}} \int_{u<\nu /|t|} u^{2} \lambda(\mathrm{d} u)+\frac{B \sigma(r)^{2} t^{2}}{2 \nu^{2}}=H(t / \nu, r) .
$$

Therefore, for $j \geq 0$,

$$
\begin{aligned}
\int\left|t^{j} \psi(t)\right|^{2} \mathrm{~d} t & =2 \int_{0}^{\infty} t^{2 j} e^{-2 \operatorname{Re}[\Psi(t)]} \mathrm{d} t \\
& \leq 2 \int_{0}^{\nu / r} t^{2 j} e^{-D(r)^{2} t^{2} / \nu^{2}} \mathrm{~d} t+2 \int_{\nu / r}^{\infty} t^{2 j} e^{-2 H(t / \nu, r)} \mathrm{d} t \\
& \leq 2 \int_{0}^{\infty} t^{2 j} e^{-D(r)^{2} t^{2} / \nu^{2}} \mathrm{~d} t+2 \nu^{2 j+1} \int_{1 / r}^{\infty} t^{2 j} e^{-2 H(t, r)} \mathrm{d} t \\
& \leq \frac{\nu^{2 j+1} \Gamma(j+1 / 2)}{D(r)^{2 j+1}}+2 \nu^{2 j+1} \int_{1 / r}^{\infty} t^{2 j} e^{-2 H(t, r)} \mathrm{d} t=2 I_{j}(r)^{2} .
\end{aligned}
$$

Next, $\psi^{\prime}(t)=-\Psi^{\prime}(t) \psi(t)$, with

$$
\Psi^{\prime}(t)=\frac{\mathrm{i}}{\nu} \int\left(1-e^{\mathrm{i} t u / \nu}\right) u\left[A \lambda_{r}(\mathrm{~d} u)+B \gamma_{r}(\mathrm{~d} u)\right]+\frac{M t}{\nu^{2}} .
$$

By $\left|1-e^{\mathrm{i} x}\right| \leq|x|$ for all $x \in \mathbb{R}$,

$$
\begin{aligned}
\left|\Psi^{\prime}(t)\right| & \leq \frac{t}{\nu^{2}} \int u^{2}\left[A \lambda_{r}(\mathrm{~d} u)+B \gamma_{r}(\mathrm{~d} u)\right]+\frac{M t}{\nu^{2}} \\
& =\frac{A \kappa_{2, X_{r}} t}{\nu^{2}}+\frac{B \kappa_{2, Y_{r}} t}{\nu^{2}}+\frac{\left(\epsilon^{2}+B \sigma(r)^{2}\right) t}{\nu^{2}} \\
& =\frac{\left(A \kappa_{2, X_{r}}+B \kappa_{2, T_{r}}\right) t}{\nu^{2}}+\frac{\epsilon^{2} t}{\nu^{2}}=\left(1+\epsilon^{2} / \nu^{2}\right) t .
\end{aligned}
$$

As a result,

$$
\begin{aligned}
\int\left|t^{j} \psi^{\prime}(t)\right|^{2} \mathrm{~d} t & =\int\left|t^{j} \Psi^{\prime}(t) \psi(t)\right|^{2} \mathrm{~d} t \\
& \leq\left(1+\epsilon^{2} / \nu^{2}\right)^{2} \int\left|t^{j+1} \psi(t)\right|^{2} \mathrm{~d} t \leq 2\left(1+\epsilon^{2} / \nu^{2}\right)^{2} I_{j+1}(r)^{2} .
\end{aligned}
$$

The proof is complete by combining Lemma 7, (42) and (43). 


\subsection{Lemmas for multivariate case}

In this section, we prove Lemmas $4-6$. Recall that $X^{*}=A_{\tau}^{-1} X_{\tau}$ and $Y^{*}=A_{\tau}^{-1} Y_{\tau}$ have Lévy measures $\lambda^{*}(\mathrm{~d} u, \mathrm{~d} \theta)=\lambda_{\tau}(\mathrm{d} v, \mathrm{~d} \omega)$ and $\gamma^{*}(\mathrm{~d} u, \mathrm{~d} \theta)=\gamma_{\tau}(\mathrm{d} v, \mathrm{~d} \omega)$, respectively, where $(v, \omega)=J_{A_{\tau}}^{-1}(u, \theta)$, i.e. $v=\left\|A_{\tau} \theta\right\| u, \omega=A_{\tau} \theta /\left\|A_{\tau} \theta\right\|$. Note that

$$
v \omega=u A_{\tau} \theta, \quad\left\|A_{\tau}^{-1} \omega\right\|=\frac{1}{\left\|A_{\tau} \theta\right\|} .
$$

Then

$$
\lambda^{*}(\mathrm{~d} u, \mathrm{~d} \theta)=\mathbf{1}\left\{v<r_{\tau}(\omega)\right\} \lambda(\mathrm{d} v \mid \omega) \nu(\mathrm{d} \omega)=\mathbf{1}\left\{u<r^{*}(\theta)\right\} \lambda^{*}(\mathrm{~d} u \mid \theta) \nu^{*}(\mathrm{~d} \theta),
$$

where

$$
r^{*}(\theta)=r_{\tau}(\omega) /\left\|A_{\tau} \theta\right\|=\left\|A_{\tau}^{-1} \omega\right\| r_{\tau}(\omega), \quad \lambda^{*}(\mathrm{~d} u \mid \theta)=\lambda(\mathrm{d} v \mid \omega), \quad \nu^{*}(\mathrm{~d} \theta)=\nu(\mathrm{d} \omega) .
$$

Similarly,

$$
\begin{aligned}
\gamma^{*}(\mathrm{~d} u, \mathrm{~d} \theta) & =m_{\tau}(\omega) \mathbf{1}\{v>0\} v^{p_{\tau}(\omega)} e^{-v / s_{\tau}(\omega)} \mathrm{d} v \nu(\mathrm{d} \omega) \\
& =m_{\tau}(\omega) \mathbf{1}\{u>0\}\left\|A_{\tau} \theta\right\|^{p_{\tau}(\omega)+1} u^{p_{\tau}(\omega)} e^{-\left\|A_{\tau} \theta\right\| u / s_{\tau}(\omega)} \mathrm{d} u \nu(\mathrm{d} \omega) \\
& =m^{*}(\theta) \mathbf{1}\{u>0\} u^{p^{*}(\theta)} e^{-u / s^{*}(\theta)} \mathrm{d} u \nu^{*}(\mathrm{~d} \theta)
\end{aligned}
$$

where

$$
m^{*}(\theta)=m_{\tau}(\omega)\left\|A_{\tau} \theta\right\|^{p_{\tau}(\omega)+1}, \quad p^{*}(\theta)=p_{\tau}(\omega), \quad s^{*}(\theta)=s_{\tau}(\omega) /\left\|A_{\tau} \theta\right\|=\left\|A_{\tau}^{-1} \omega\right\| s_{\tau}(\omega) .
$$

Therefore,

$$
\gamma^{*}(\mathrm{~d} u, \mathrm{~d} \theta)=\gamma^{*}(\mathrm{~d} u \mid \theta) \nu^{*}(\theta), \text { with } \gamma^{*}(\mathrm{~d} u \mid \theta)=m^{*}(\theta) \mathbf{1}\{u>0\} u^{p^{*}(\theta)} e^{-u / s^{*}(\theta)} \mathrm{d} u .
$$

Lemma 8. If (24) holds under $\nu$, then

$$
\operatorname{essinf} p^{*}(\theta)>-2, \quad \operatorname{ess} \sup \frac{\left[p^{*}(\theta)+3\right] s^{*}(\theta)}{r^{*}(\theta)} \leq 1, \quad \text { under } \nu^{*} .
$$

Furthermore, for $R^{*}$ and $S^{*}$ in (25) defined under $\nu$, we have

$$
R^{*}=\operatorname{ess} \sup r^{*}(\theta), \quad S^{*}=\operatorname{ess} \sup \left[p^{*}(\theta)+h(d)+1\right] s^{*}(\theta), \quad \text { under } \nu^{*} .
$$

Finally, for $\varrho_{\tau}(a)$ is defined in (23), we have

$$
\begin{aligned}
\varrho_{\tau}(z) & =\text { smallest eigenvalue of } C_{1}^{2} M^{*}(z) \text { and } K^{*} \\
\text { with } M^{*}(z) & =\int u^{2} \theta \theta^{\prime} \mathbf{1}\left\{u<r^{*}(\theta) \wedge z\right\} \lambda^{*}(\mathrm{~d} u, \mathrm{~d} \theta) .
\end{aligned}
$$

Proof. The lemma is straightforward except for the assertion on $\varrho_{\tau}(a)$. By change of variable $(u, \theta)=J_{A_{\tau}}(v, \omega)$,

$$
\begin{aligned}
A_{\tau} M^{*}(z) A_{\tau} & =\int\left(u A_{\tau} \theta\right)\left(u A_{\tau} \theta\right)^{\prime} 1\left\{u<r^{*}(\theta) \wedge z\right\} \lambda^{*}(\mathrm{~d} u, \mathrm{~d} \theta) \\
& =\int(v \omega)(v \omega)^{\prime} \mathbf{1}\left\{\left\|A_{\tau}^{-1} \omega\right\| v<\left(\left\|A_{\tau}^{-1} \omega\right\| r_{\tau}(\omega)\right) \wedge z\right\} \lambda_{\tau}(\mathrm{d} v, \mathrm{~d} \omega) .
\end{aligned}
$$

Since the right hand side is $M_{\tau}(z), M^{*}(z)=A_{\tau}^{-1} M(z) A_{\tau}^{-1}$. Then the assertion on $\varrho_{\tau}(z)$ follows. 
Proof of Lemma 4. Fix $a \geq 3$. Since the Lévy measure $\lambda^{*}$ of $X^{*}$ has bounded support according to condition (25) and Lemma 8, $\mathbb{E}\left\|X^{*}\right\|^{a}<\infty$.

$$
\begin{aligned}
\int u^{a} \gamma^{*}(\mathrm{~d} u, \mathrm{~d} \theta) & =\int m^{*}(\theta) \nu(\mathrm{d} \theta) \int u^{p^{*}(\theta)+a} e^{-u / s^{*}(\theta)} \mathrm{d} u \\
& =\int m^{*}(\theta) \Gamma\left(p^{*}(\theta)+a+1\right) s^{*}(\theta)^{p^{*}(\theta)+a+1} \nu(\mathrm{d} \theta) .
\end{aligned}
$$

Letting

$$
b=\operatorname{ess} \sup \frac{\Gamma\left(p^{*}(\theta)+a+1\right) s^{*}(\theta)^{a-2}}{\Gamma\left(p^{*}(\theta)+3\right)},
$$

then, by the construction of $Y^{*}$,

$$
\begin{aligned}
\int u^{a} \gamma^{*}(\mathrm{~d} u, \mathrm{~d} \theta) & \leq b \int m^{*}(\theta) \Gamma\left(p^{*}(\theta)+3\right) s^{*}(\theta)^{p^{*}(\theta)+3} \nu(\mathrm{d} \theta) \\
& =b \int \nu(\mathrm{d} \theta) \int u^{2} \lambda_{\tau}^{*}(\mathrm{~d} u \mid \theta)=b \int u^{2} \lambda^{*}(\mathrm{~d} u, \mathrm{~d} \theta) .
\end{aligned}
$$

Since $b \leq \operatorname{ess} \sup \left[\left(p^{*}(\theta)+a\right) s^{*}(\theta)\right]^{a-2}$ and by conditions (24) and (25) as well as Lemma 8 ,

$$
\operatorname{ess} \sup \left[\left(p^{*}(\theta)+a\right) s^{*}(\theta)\right] \leq \operatorname{ess} \sup \frac{p^{*}(\theta)+a}{p^{*}(\theta)+3}<\infty,
$$

then $b<\infty$. Thus $\int u^{a} \gamma^{*}(\mathrm{~d} u, \mathrm{~d} \theta)<\infty$, giving $\mathbb{E}\left\|Y^{*}\right\|^{a}<\infty$.

Lemma 9. Given $\tau>0$, under conditions (24) and (25), $\Psi_{X^{*}}$ and $\Psi_{Y^{*}} \in C^{\infty}\left(\mathbb{R}^{d}\right)$ such that

$$
\left|\partial_{j} \Psi_{X^{*}}(t)\right| \leq d\|t\|, \quad\left|\partial_{j} \Psi_{T^{*}}(t)\right| \leq d\|t\|, \quad j=1, \ldots, d
$$

and for $|\alpha| \geq 2$,

$$
\left|\partial^{\alpha} \Psi_{X^{*}}(t)\right| \leq\left(R^{*}\right)^{|\alpha|-2} d, \quad\left|\partial^{\alpha} \Psi_{T^{*}}(t)\right| \leq\left(S^{*} \operatorname{ess} \sup \frac{p^{*}(\theta)+|\alpha|}{p^{*}(\theta)+h(d)+1}\right)^{|\alpha|-2} d .
$$

In particular, if $2 \leq|\alpha| \leq h(d)$, then $\left|\partial^{\alpha} \Psi_{T^{*}}(t)\right| \leq\left(S^{*}\right)^{|\alpha|-2} d$.

Proof. By Lemma 4, $\int\|u\|^{a} \lambda^{*}(\mathrm{~d} u, \mathrm{~d} \theta)<\infty$ for $a \geq 2$, so by dominated convergence, $\Psi_{X^{*}} \in$ $C^{\infty}\left(\mathbb{R}^{d}\right), \partial_{j} \Psi_{X^{*}}(t)=\int \mathrm{i} u \theta_{j}\left(1-e^{\mathrm{i} u\langle t, \theta\rangle}\right) \lambda^{*}(\mathrm{~d} u, \mathrm{~d} \theta)$, and $\partial^{\alpha} \Psi_{X^{*}}(t)=-\int(\mathrm{i} u \theta)^{\alpha} e^{\mathrm{i} u\langle t, \theta\rangle} \lambda^{*}(\mathrm{~d} u, \mathrm{~d} \theta)$ for $|\alpha| \geq 2$. For $\Psi_{Y^{*}}$, similar formulas hold. From $\left|\partial_{j} \Psi_{X^{*}}(t)\right| \leq\|t\| \int u^{2} \lambda^{*}(\mathrm{~d} u, \mathrm{~d} \theta)=\|t\| \operatorname{tr}\left(\mathbb{V}\left(X^{*}\right)\right)$, the first inequality follows. Likewise, $\left|\partial_{j} \Psi_{Y^{*}}(t)\right| \leq \| t|| \operatorname{tr}\left(\mathbb{V}\left(Y^{*}\right)\right)$. Together with $\frac{1}{2}\left|\partial_{j}\left(t^{\prime} K^{*} t\right)\right| \leq$ $\left\|K^{*} t\right\| \leq\|t\| \operatorname{tr}\left(K^{*}\right)$, this implies the second inequality. Next, for $|\alpha| \geq 2$, from

$$
\begin{aligned}
\left|\partial^{\alpha} \Psi_{X^{*}}(t)\right| & \leq \int u^{|\alpha|} \mathbf{1}\left\{u \leq r^{*}(\theta)\right\} \lambda^{*}(\mathrm{~d} u, \mathrm{~d} \theta) \\
& \leq\left(R^{*}\right)^{|\alpha|-2} \int u^{2} \lambda^{*}(\mathrm{~d} u, \mathrm{~d} \theta)=\left(R^{*}\right)^{|\alpha|-2} \operatorname{tr}\left(\mathbb{V}\left(X^{*}\right)\right),
\end{aligned}
$$

the third inequality follows. Finally, for $|\alpha| \geq 2$, let

$$
C=\operatorname{ess} \sup \left[s^{*}(\theta)^{|\alpha|-2} \frac{\Gamma\left(p^{*}(\theta)+|\alpha|+1\right)}{\Gamma\left(p^{*}(\theta)+3\right)}\right] .
$$


Then by Lemma 8 ,

$$
\begin{aligned}
\left|\partial^{\alpha} \Psi_{Y^{*}}(t)\right| & \leq \int \nu^{*}(\mathrm{~d} \theta) \int_{0}^{\infty} u^{|\alpha|} \gamma^{*}(\mathrm{~d} u \mid \theta) \\
& =\int m^{*}(\theta)\left[s^{*}(\theta)\right]^{p^{*}(\theta)+|\alpha|+1} \Gamma\left(p^{*}(\theta)+|\alpha|+1\right) \nu^{*}(\mathrm{~d} \theta) \\
& \leq C \int m^{*}(\theta)\left[s^{*}(\theta)\right]^{p^{*}(\theta)+3} \Gamma\left(p^{*}(\theta)+3\right) \nu^{*}(\mathrm{~d} \theta) \\
& =C \int u^{2} \gamma^{*}(\mathrm{~d} u, \mathrm{~d} \theta)=C \operatorname{tr}\left(\mathbb{V}\left(Y^{*}\right)\right) .
\end{aligned}
$$

Since $|\alpha| \geq 2$, then by (25) and Lemma 8 ,

$$
C \leq\left[\operatorname{ess} \sup s^{*}(\theta)\left(p^{*}(\theta)+|\alpha|\right)\right]^{|\alpha|-2} \leq\left(S^{*} \operatorname{ess} \sup \frac{p^{*}(\theta)+|\alpha|}{p^{*}(\theta)+h(d)+1}\right)^{|\alpha|-2} .
$$

Also, $\left|\partial^{\alpha} \Psi_{Z^{*}}(t)\right| \leq \operatorname{tr}\left(K^{*}\right) \mathbf{1}\{|\alpha|=2\}$. We therefore get the last inequality.

Lemma 10. Let $C_{1}$ and $C_{2}$ be the same constants defined in Section 3.2. Given $\tau>0$, under conditions (24) and (25), the following statements are true.

1) If $\|t\| \leq 1 / R^{*}$, then

$$
\operatorname{Re}\left[\Psi_{X^{*}}(t)\right] \geq \frac{C_{1}^{2}\|t\|^{2}}{2}, \quad \operatorname{Re}\left[\Psi_{T^{*}}(t)\right] \geq \frac{C_{1}^{2} C_{2}\|t\|^{2}}{2} .
$$

2) If $\|t\|>1 / R^{*}$, then

$$
\operatorname{Re}\left[\Psi_{X^{*}}(t)\right] \geq \frac{\varrho_{\tau}(1 /\|t\|)\|t\|^{2}}{2}, \quad \operatorname{Re}\left[\Psi_{T^{*}}(t)\right] \geq \frac{t^{\prime} K^{*} t}{2} .
$$

Proof. 1) Given $\|t\| \leq 1 / R^{*}$, by Lemma 8, for $\nu^{*}$-a.e. $\theta \in S$ and $0<u<r^{*}(\theta),|\langle t, \theta\rangle u| \leq$ $|\langle t, \theta\rangle| r^{*}(\theta) \leq 1$, yielding $1-\cos (\langle t, \theta\rangle u) \geq C_{1}^{2}\langle t, \theta\rangle^{2} u^{2} / 2$. Therefore,

$$
\begin{aligned}
\operatorname{Re}\left[\Psi_{X^{*}}(t)\right] & =\int[1-\cos (\langle t, \theta\rangle u)] \mathbf{1}\left\{u<r^{*}(\theta)\right\} \lambda^{*}(\mathrm{~d} u \mid \theta) \nu^{*}(\mathrm{~d} \theta) \\
& \geq \frac{C_{1}^{2}}{2} \int\langle t, \theta\rangle^{2} u^{2} \mathbf{1}\left\{u<r^{*}(\theta)\right\} \lambda^{*}(\mathrm{~d} u \mid \theta) \nu^{*}(\mathrm{~d} \theta)=\frac{C_{1}^{2}}{2} \int\langle t, \theta\rangle^{2} u^{2} \lambda^{*}(\mathrm{~d} u, \mathrm{~d} \theta) .
\end{aligned}
$$

Since the last integral equals $t^{\prime} \mathbb{V}\left(X^{*}\right) t=\|t\|^{2}$, we get the first inequality in 1$)$. Next, by (25) and Lemma 8, for $\nu^{*}$-a.e. $\theta \in S$ and $0<u \leq s^{*}(\theta)\left[p^{*}(\theta)+3\right],|\langle t, \theta\rangle u| \leq\|t\| u \leq r^{*}(\theta) / R^{*} \leq 1$. Then

$$
\begin{aligned}
\int_{0}^{\infty}[1-\cos (\langle t, \theta\rangle u)] \gamma^{*}(\mathrm{~d} u \mid \theta) & \geq \int_{0}^{s^{*}(\theta)\left[p^{*}(\theta)+3\right]}[1-\cos (\langle t, \theta\rangle u)] \gamma^{*}(\mathrm{~d} u \mid \theta) \\
& \geq \frac{C_{1}^{2}}{2} \int_{0}^{s^{*}(\theta)\left[p^{*}(\theta)+3\right]}\langle t, \theta\rangle^{2} u^{2} \gamma^{*}(\mathrm{~d} u \mid \theta) \\
& \geq \frac{C_{1}^{2} C_{2}}{2} \int_{0}^{\infty}\langle t, \theta\rangle^{2} u^{2} \gamma^{*}(\mathrm{~d} u \mid \theta) .
\end{aligned}
$$

Then

$$
\begin{aligned}
\operatorname{Re}\left[\Psi_{Y^{*}}(t)\right] & =\int[1-\cos (\langle t, \theta\rangle u)] \gamma^{*}(\mathrm{~d} u \mid \theta) \nu^{*}(\mathrm{~d} \theta) \\
& \geq \frac{C_{1}^{2} C_{2}}{2} \int\langle t, \theta\rangle^{2} u^{2} \gamma^{*}(\mathrm{~d} u, \mathrm{~d} \theta)=\frac{C_{1}^{2} C_{2}}{2} t^{\prime} \mathbb{V}\left(Y^{*}\right) t
\end{aligned}
$$


and hence

$$
\begin{aligned}
\operatorname{Re}\left[\Psi_{T^{*}}(t)\right] & =\operatorname{Re}\left[\Psi_{Y^{*}}(t)\right]+\operatorname{Re}\left[\Psi_{Z^{*}}(t)\right] \\
& \geq \frac{C_{1}^{2} C_{2}}{2} t^{\prime} \mathbb{V}\left(Y^{*}\right) t+\frac{1}{2} t^{\prime} K^{*} t \geq \frac{C_{1}^{2} C_{2}}{2} t^{\prime}\left[\mathbb{V}\left(Y^{*}\right)+K^{*}\right] t
\end{aligned}
$$

Since $\mathbb{V}\left(Y^{*}\right)+K^{*}=\mathbb{V}\left(T^{*}\right)=I$, then the second inequality in 1) follows.

2) The first inequality follows from

$$
\begin{aligned}
\operatorname{Re}\left[\Psi_{X^{*}}(t)\right] & \geq \int[1-\cos (\langle t, \theta\rangle u)] \mathbf{1}\left\{u<r^{*}(\theta) \wedge \frac{1}{\|t\|}\right\} \lambda^{*}(\mathrm{~d} u, \mathrm{~d} \theta) \\
& \geq \frac{C_{1}^{2}}{2} \int\langle t, \theta\rangle^{2} u^{2} \mathbf{1}\left\{u<r^{*}(\theta) \wedge \frac{1}{\|t\|}\right\} \lambda^{*}(\mathrm{~d} u, \mathrm{~d} \theta)=\frac{t^{\prime} M^{*}(1 /\|t\|) t}{2}
\end{aligned}
$$

and Lemma 8, while the second one from $\operatorname{Re}\left[\Psi_{T^{*}}(t)\right] \geq \operatorname{Re}\left[\Psi_{Z^{*}}(t)\right]=t^{\prime} K^{*} t / 2$.

Proof of Lemma 5. The proof for 1) is completely similar to that for 1) of Lemma 1, except that it is based on Lemma 9. To prove 2), let $k=h(d)$. By Cauchy-Schwartz inequality,

$$
\int\|t\|^{q}\left|\psi_{X^{*}}(t)\right| \mathrm{d} t \leq\left(\int\|t\|^{2 q}\left(1+\|t\|^{2 k}\right)\left|\psi_{X^{*}}(t)\right|^{2} \mathrm{~d} t\right)^{1 / 2}\left(\int \frac{\mathrm{d} t}{1+\|t\|^{2 k}}\right)^{1 / 2} .
$$

The second factor on the right hand side is finite. By 2) of Lemma 10, for $\|t\|>1 / R^{*},\left|\psi_{X^{*}}(t)\right| \leq$ $\exp \left\{-\operatorname{Re}\left[\Psi_{X^{*}}(t)\right]\right\} \leq \exp \left\{-\varrho_{\tau}(1 /\|t\|)\|t\|^{2} / 2\right\}$. Then

$$
\begin{aligned}
\int_{\|t\|>1 / R^{*}}\|t\|^{2 q}\left(1+\|t\|^{2 k}\right)\left|\psi_{X^{*}}(t)\right|^{2} \mathrm{~d} t & \leq \int_{\|t\|>1 / R^{*}}\|t\|^{2 q}\left(1+\|t\|^{2 k}\right) e^{-\varrho_{\tau}(1 /\|t\|)\|t\|^{2}} \mathrm{~d} t \\
& \leq c(d) \int s^{2 q+d-1}\left(1+s^{2 k}\right) e^{-\varrho_{\tau}(1 / s) s^{2}} \mathrm{~d} s,
\end{aligned}
$$

where $c(d)$ is a universal constant. Then by condition (26), $\|t\|^{q}\left|\psi_{X^{*}}(t)\right| \in L^{1}\left(\mathbb{R}^{d}\right)$ and the proof follows from Proposition 28.1 of [31].

To prove Lemma 6, we use the following variant of Lemma 11.6 of [5] which involves lower order of partial derivatives.

Lemma 11. For $m \geq 1$, there is a constant $c_{1}(d, m)$, such that for $f \in \mathscr{S}\left(\mathbb{R}^{d}\right)$,

$$
\max _{|\alpha|=m} \int\left|f^{(\alpha)}(x)\right| \mathrm{d} x \leq c_{1}(d, m) \max \left\{\sqrt{\int\left|t^{\beta} \partial_{i}^{j} \widehat{f}(t)\right|^{2} \mathrm{~d} t}:|\beta| \leq m, 1 \leq i \leq d, 0 \leq j \leq h(d)\right\} .
$$

Proof. Denote $k=h(d)$ and $w(x)=x_{1}^{2 k}+\cdots+x_{n}^{2 k}$. By Cauchy-Schwartz inequality,

$$
\int\left|f^{(\alpha)}(x)\right| \mathrm{d} x \leq \sqrt{\int \frac{\mathrm{d} x}{1+w(x)}} \sqrt{\int\left|f^{(\alpha)}(x)\right|^{2}(1+w(x)) \mathrm{d} x} .
$$

First,

$$
\int \frac{\mathrm{d} x}{1+w(x)} \leq d \int \frac{\mathrm{d} x_{1}}{1+x_{1}^{2 k}} \int_{0 \leq\left|x_{i}\right| \leq\left|x_{1}\right|} \mathrm{d} x_{2} \cdots \mathrm{d} x_{k}=d 2^{d} \int_{0}^{\infty} \frac{x^{d-1} \mathrm{~d} x}{1+x^{2 k}}=c^{\prime}(d) .
$$


Next, by Plancherel theorem and properties of the Fourier transform ([18], p. 100-102),

$$
\begin{aligned}
\int\left|f^{(\alpha)}(x)\right|^{2}(1+w(x)) \mathrm{d} x & =\int\left|f^{(\alpha)}(x)\right|^{2} \mathrm{~d} x+\sum_{i=1}^{d} \int\left|x_{i}^{k} f^{(\alpha)}(x)\right|^{2} \mathrm{~d} x \\
& =\int\left|t^{\alpha} \widehat{f}(t)\right|^{2} \mathrm{~d} t+\sum_{i=1}^{d} \int\left|\partial_{i}^{k}\left[t^{\alpha} \widehat{f}(t)\right]\right|^{2} \mathrm{~d} t
\end{aligned}
$$

so by $\sqrt{a+b} \leq \sqrt{a}+\sqrt{b}$ for $a, b \geq 0$,

$$
\sqrt{\int\left|f^{(\alpha)}(x)\right|^{2}(1+w(x)) \mathrm{d} x} \leq \sqrt{\int\left|t^{\alpha} \widehat{f}(t)\right|^{2} \mathrm{~d} t}+\sum_{i=1}^{d} \sqrt{\int\left|\partial_{i}^{k}\left[t^{\alpha} \widehat{f}(t)\right]\right|^{2} \mathrm{~d} t} .
$$

Since for each $i=1, \ldots, d$,

$$
\begin{aligned}
\partial_{i}^{k}\left[t^{\alpha} \widehat{f}(t)\right] & =\sum_{0 \leq j \leq \alpha_{i} \wedge k}\left(\begin{array}{l}
k \\
j
\end{array}\right) \partial_{i}^{j} t^{\alpha} \cdot \partial_{i}^{k-j} \widehat{f}(t) \\
& =\sum_{0 \leq j \leq \alpha_{i} \wedge k}\left(\begin{array}{l}
k \\
j
\end{array}\right) \frac{\alpha_{i} !}{\left(\alpha_{i}-j\right) !} t^{\alpha} t_{i}^{-j} \partial_{i}^{k-j} \widehat{f}(t),
\end{aligned}
$$

by Minkowski inequality, the desired inequality follows.

Finally, notice that for $k \geq 1$, there is a unique multivariate polynomial of $x=\left(x_{1}, \ldots, x_{k}\right) \in \mathbb{C}^{k}$,

$$
P_{k}(x)=\sum_{i \in I_{k}} a_{i} x^{i}, \quad \text { with } a_{i} \in \mathbb{Z}_{+},
$$

where $I_{k}=\left\{i=\left(i_{1}, \ldots, i_{k}\right) \in \mathbb{Z}_{+}^{k}: \sum_{j=1}^{k} j i_{j}=k\right\}$, such that for any $k$-times differentiable function $\Psi$ on $\mathbb{R}$, letting $\psi=\exp (\Psi)$,

$$
\psi^{(k)}=P_{k}\left(\Psi^{\prime}, \Psi^{\prime \prime}, \ldots, \Psi^{(k)}\right) \psi=\sum_{i \in I_{k}} a_{i_{1} i_{2} \cdots i_{k}}\left(\Psi^{\prime}\right)^{i_{1}}\left(\Psi^{\prime \prime}\right)^{i_{2}} \cdots\left(\Psi^{(k)}\right)^{i_{k}} \psi
$$

Note that if $\Psi(t)=-t^{2} / 2$, then $(-1)^{k} P_{k}\left(\Psi^{\prime}(t), \ldots, \Psi^{(k)}(t)\right)$ is the $k$ th-order Hermite polynomial. Proof of Lemma 6. Denote $k=h(d)$. By Lemma $5, f_{\xi} \in \mathscr{S}\left(\mathbb{R}^{d}\right)$. By Lemma 9 , for $i=1, \ldots, d$

$$
\left|\partial_{i} \Psi_{\xi}(t)\right| \leq A\left|\partial_{i} \Psi_{X^{*}}(t)\right|+B\left|\partial_{i} \Psi_{T^{*}}(t)\right|+\epsilon^{2}\|t\| \leq\left(d+\epsilon^{2}\right)\|t\|
$$

and for $2 \leq j \leq k$,

$$
\begin{aligned}
\left|\partial_{i}^{j} \Psi_{\xi}(t)\right| & \leq A\left|\partial_{i}^{j} \Psi_{X^{*}}(t)\right|+B\left|\partial_{i}^{j} \Psi_{T^{*}}(t)\right|+\epsilon^{2} \mathbf{1}\{j=2\} \\
& \leq\left(R^{*}\right)^{j-2} A d+\left(S^{*}\right)^{j-2} B d+\epsilon^{2} \mathbf{1}\{j=2\} \\
& \leq A d+B d+\epsilon^{2} \mathbf{1}\{j=2\} \leq d+\epsilon^{2}
\end{aligned}
$$

Thus, by (44), $\left|\partial_{i}^{j} \psi_{\xi}(t)\right| \leq\left(d+\epsilon^{2}\right)^{j} P_{j}(\|t\|, 1,1, \ldots, 1)\left|\psi_{\xi}(t)\right|$. Since $P_{j}(x, 1, \ldots, 1)$ is a $j$ th-order polynomial of $x \in \mathbb{C}$ with coefficients only depending on $j$, there is a polynomial $h(x)=h_{d, m}(x)$ of 
order no greater than $2 m+2 k$ with coefficients only depending on $(d, m)$, such that for all $\beta$ with $|\beta| \leq m, i=1, \ldots, d$ and $0 \leq j \leq k$,

$$
\int\left|t^{\beta} \partial_{i}^{j} \psi_{\xi}(t)\right|^{2} \mathrm{~d} t \leq\left(d+\epsilon^{2}\right)^{2 k} \int h(\|t\|)\left|\psi_{\xi}(t)\right|^{2} \mathrm{~d} t
$$

Write

$$
I_{1}=\int \mathbf{1}\left\{\|t\| \leq 1 / R^{*}\right\} h(\|t\|)\left|\psi_{\xi}(t)\right|^{2} \mathrm{~d} t, \quad I_{2}=\int \mathbf{1}\left\{\|t\|>1 / R^{*}\right\} h(\|t\|)\left|\psi_{\xi}(t)\right|^{2} \mathrm{~d} t .
$$

By 1) of Lemma 10, for $\|t\| \leq 1 / R^{*}$,

$$
\operatorname{Re}\left[\Psi_{\xi}(t)\right] \geq A \operatorname{Re}\left[\Psi_{X^{*}}(t)\right]+B \operatorname{Re}\left[\Psi_{T^{*}}(t)\right] \geq C_{1}^{2} C_{2}\|t\|^{2} / 2 .
$$

Then

$$
I_{1} \leq \int h(\|t\|) \exp \left\{-2 \operatorname{Re}\left[\Psi_{\xi}(t)\right]\right\} \mathrm{d} t \leq \int h(\|t\|) \exp \left\{-C_{1}^{2} C_{2}\|t\|^{2}\right\} \mathrm{d} t=c^{\prime}(d, m) .
$$

On the other hand, by 2) of Lemma 10 , for $\|t\|>1 / R^{*}$,

$$
\operatorname{Re}\left[\Psi_{\xi}(t)\right] \geq A \operatorname{Re}\left[\Psi_{X^{*}}(t)\right]+B \operatorname{Re}\left[\Psi_{T^{*}}(t)\right] \geq \varrho_{\tau}(1 /\|t\|)\|t\|^{2} / 2 .
$$

Therefore, by change of variable $t=s \omega$ with $s>0$ and $\omega \in S$,

$$
\begin{aligned}
I_{2} & \leq \int \mathbf{1}\left\{\|t\|>1 / R^{*}\right\} h(\|t\|) \exp \left(-\varrho_{\tau}(1 /\|t\|)\|t\|^{2}\right) \mathrm{d} t \\
& =c^{\prime \prime}(d) \int_{1 / R^{*}}^{\infty} s^{d-1} h(s) e^{-\varrho_{\tau}(1 / s) s^{2}} \mathrm{~d} s .
\end{aligned}
$$

As a result, for all $\beta$ with $|\beta| \leq m, i=1, \ldots, d$ and $0 \leq j \leq k$,

$$
\int\left|t^{\beta} \partial_{i}^{j} \psi_{\xi}(t)\right|^{2} \mathrm{~d} t \leq\left(1+\epsilon^{2} / d\right)^{2 k} d^{2 k}\left(c^{\prime}(d, m)+c^{\prime \prime}(d) \int_{1 / R^{*}}^{\infty} s^{d-1} h(s) e^{-\varrho_{\tau}(1 / s) s^{2}} \mathrm{~d} s\right) .
$$

Combining the bound with Lemma 11, the proof is complete.

\section{References}

[1] Asmussen, S. And Rosiński, J. (2001). Approximations of small jumps of Lévy processes with a view towards simulation. J. Appl. Probab. 38, 2, 482-493.

[2] Baeumer, B. And Kovács, M. (2012). Approximating multivariate tempered stable processes. J. Appl. Probab. 49, 1, 167-183.

[3] Barbour, A. D. and Chen, L. H. Y. (2005). The permutation distribution of matrix correlation statistics. In Stein's method and applications. Lect. Notes Ser. Inst. Math. Sci. Natl. Univ. Singap., Vol. 5. Singapore Univ. Press, Singapore, 223-245.

[4] Bertoin, J. (1996). Lévy processes. Cambridge Tracts in Mathematics, Vol. 121. Cambridge University Press, Cambridge. 
[5] Bhattacharya, R. N. And Ranga Rao, R. (1976). Normal approximation and asymptotic expansions. John Wiley \& Sons, New York-London-Sydney. Wiley Series in Probability and Mathematical Statistics.

[6] Chatterjee, S. (2006). A generalization of the Lindeberg principle. Ann. Probab. 34, 6, 2061-2076.

[7] Chatterjee, S. And Shao, Q.-M. (2011). Nonnormal approximation by Stein's method of exchangeable pairs with application to the Curie-Weiss model. Ann. Appl. Probab. 21, 2, 464-483.

[8] Chen, L. H. Y., Goldstein, L., And Shao, Q.-M. (2011). Normal approximation by Stein's method. Probability and its Applications (New York). Springer, Heidelberg.

[9] CHI, Z. (2012). On exact sampling of nonnegative infinitely divisible random variables. Adv. Appl. Probab. 44, 3, 842-873.

[10] Chow, Y. S. And Teicher, H. (1997). Probability theory: independence, interchangeability, martingales, 3 ed. Springer Texts in Statistics. Springer-Verlag, New York.

[11] Cohen, S. And Rosiński, J. (2007). Gaussian approximation of multivariate Lévy processes with applications to simulation of tempered stable processes. Bernoulli 13, 1, 195-210.

[12] Daley, D. J. And Vere-Jones, D. (2002). An introduction to the theory of point processes, 2 ed.Vol. 1. . Springer-Verlag, New York.

[13] Dereich, S. (2011). Multilevel Monte Carlo algorithms for Lévy-driven SDEs with Gaussian correction. Ann. Appl. Probab. 21, 1, 283-311.

[14] Devroye, L. (1986). Nonuniform random variate generation. Springer-Verlag, New York.

[15] Devroye, L. (1987). A simple generator for discrete log-concave distributions. Computing 39, 1, 87-91.

[16] Fournier, N. (2011). Simulation and approximation of Lévy-driven stochastic differential equations. ESAIM Probab. Stat. 15, 233-248.

[17] Götze, F. (1991). On the rate of convergence in the multivariate CLT. Ann. Probab. 19, 2, $724-739$.

[18] Grafakos, L. (2008). Classical Fourier analysis, 2nd ed. Graduate Texts in Mathematics, Vol. 249. Springer, New York.

[19] Hilber, N., Reich, N., Schwab, C., And Winter, C. (2009). Numerical methods for Lévy processes. Finance Stoch. 13, 4, 471-500.

[20] Hörmann, W., Leydold, J., And Derflinger, G. (2004). Automatic nonuniform random variate generation. Statistics and Computing. Springer-Verlag, Berlin.

[21] Kallenberg, O. (2002). Foundations of modern probability, Second ed. Probability and its Applications (New York). Springer-Verlag, New York.

[22] Kawai, R. And Masuda, H. (2011). On simulation of tempered stable random variates. $J$. Comput. Appl. Math. 235, 8, 2873-2887. 
[23] Kohatsu-Higa, A. And Tankov, P. (2010). Jump-adapted discretization schemes for Lévydriven SDEs. Stochastic Processes Appl. 120, 11, 2258-2285.

[24] Korevane, J. (2004). Tauberian theory. Grundlehren der Mathematischen Wissenschaften [Fundamental Principles of Mathematical Sciences], Vol. 329. Springer-Verlag, Berlin. A century of developments.

[25] Ledoux, M. and Talagrand, M. (1991). Probability in Banach spaces. Ergebnisse der Mathematik und ihrer Grenzgebiete (3) [Results in Mathematics and Related Areas (3)], Vol. 23. Springer-Verlag, Berlin. Isoperimetry and processes.

[26] Lorz, U. And Heinrich, L. (1991). Normal and Poisson approximation of infinitely divisible distribution functions. Statistics 22, 4, 627-649.

[27] Nourdin, I. And Peccati, G. (2009). Stein's method on Wiener chaos. Probab. Theory Related Fields 145, 1-2, 75-118.

[28] Nourdin, I. And Peccati, G. (2012). Normal approximations with Malliavin calculus. Cambridge Tracts in Mathematics, Vol. 192. Cambridge University Press, Cambridge. From Stein's method to universality.

[29] Orey, S. (1968). On continuity properties of infinitely divisible distribution functions. Ann. Math. Stat. 39, 936-937.

[30] Reinert, G. (2005). Three general approaches to Stein's method. In An introduction to Stein's method. Lect. Notes Ser. Inst. Math. Sci. Natl. Univ. Singap., Vol. 4. Singapore Univ. Press, Singapore, 183-221.

[31] Sato, K.-I. (1999). Lévy processes and infinitely divisible distributions. Cambridge Studies in Advanced Mathematics, Vol. 68. Cambridge University Press, Cambridge. Translated from the 1990 Japanese original, Revised by the author.

[32] Sazonov, V. V. (1968). On the multi-dimensional central limit theorem. Sankhyā Ser. A 30, $181-204$.

[33] Veillette, M. S. and Taqqu, M. S. (2012). Berry-Esseen and Edgeworth approximations for the normalized tail of an infinite sum of independent weighted gamma random variables. Stochastic Processes Appl. 122, 3, 885-909. 